\title{
THE MEAN SQUARE OF THE PRODUCT OF A DIRICHLET $L$-FUNCTION AND A DIRICHLET POLYNOMIAL
}

\author{
B. CONREY, H. IWANIEC, AND K. SOUNDARARAJAN
}

\section{INTRODUCTION}

This paper is motivated by work of Balasubramanian, Conrey, and Heath-Brown [4] who established that

$$
\int_{0}^{T}|A(1 / 2+i t) \zeta(1 / 2+i t)|^{2} d t=T \sum_{h, k \leq T^{\vartheta}} \frac{\lambda_{h} \overline{\lambda_{k}}(h, k)}{h k}\left(\log \frac{T(h, k)^{2}}{2 \pi h k}+2 \gamma-1\right)+o(T)
$$

where

$$
A(s)=\sum_{h \leq T^{\vartheta}} \frac{\lambda_{h}}{h^{s}}
$$

is a Dirichlet polynomial with length $T^{\vartheta}$ for any fixed $\vartheta<1 / 2$, and the coefficients $\lambda_{h}$ satisfy $\lambda_{h} \ll h^{\epsilon}$. In [4], it was conjectured that (1) holds for longer Dirichlet polynomials $A$, again with coefficients $\lambda_{h} \ll h^{\epsilon}$, and with length $T^{\vartheta}$ with any fixed $\vartheta<1$. This conjecture implies the Lindelöf hypothesis, and also that at least $3 / 5$ of the zeros of the Riemann zeta-function are on the critical line. Recent work of Bettin, Chandee, and Radziwill [3] establishes (1) for Dirichlet polynomials of length $T^{\vartheta}$ provided $\vartheta<\frac{1}{2}+0.01515$.

One can formulate a more general version of this conjecture, by introducing "shifts." Let $T$ be large, and suppose $\alpha$ and $\beta$ are two small complex numbers both $\ll 1 / \log T$. Let $h$ and $k$ be natural numbers, and $\psi$ a fixed compactly supported smooth function on $\mathbb{R}^{+}$. Then, generalizing (11), one may conjecture that

$$
\begin{aligned}
& \int_{0}^{\infty} \psi\left(\frac{t}{T}\right) \zeta\left(\frac{1}{2}+i t+\alpha\right) \zeta\left(\frac{1}{2}-i t+\beta\right)(h / k)^{i t} d t \\
& =\int_{0}^{\infty} \psi\left(\frac{t}{T}\right) \\
& \begin{aligned}
& \left(\frac{(h, k)^{1+\alpha+\beta}}{h^{1 / 2+\beta} k^{1 / 2+\alpha}} \zeta(1+\alpha+\beta)+\left(\frac{t}{2 \pi}\right)^{-\alpha-\beta} \frac{(h, k)^{1-\alpha-\beta}}{h^{1 / 2-\alpha} k^{1 / 2-\beta}} \zeta(1-\alpha-\beta)\right) d t \\
& +E_{h, k}(T)
\end{aligned}
\end{aligned}
$$

The first author is partially supported by the NSF, and by a Programme grant from the EPSRC. The second author is partially supported by the NSF grant DMS 1406981. The third author is partially supported by the NSF, and a Simons Investigator grant from the Simons Foundation. 
where the remainder terms $E_{h, k}(T)$ satisfy

$$
\sum_{h, k \leq T^{\vartheta}} \frac{\lambda_{h} \overline{\lambda_{k}}}{\sqrt{h k}} E_{h, k}(T)=o(T)
$$

provided the coefficients $\lambda_{h}$ are $\ll h^{\epsilon}$, and $\vartheta<1$. When $\alpha+\beta=0$, the main term in (2) should be interpreted in the sense of a limit. The introduction of the shifts $\alpha$ and $\beta$ permits the main terms to be expressed in a more transparent way, and also allows (via Cauchy's formula) one to deduce variants for derivatives of $\zeta(s)$.

In this paper we consider analogues of the conjectures (11) and (2) in the context of Dirichlet $L$-functions to a large modulus $q$. It has long been known that such $q$-analogues behave similarly to the situation of the zeta-function in $t$-aspect (for example, see Selberg [13], Iwaniec and Sarnak [12, Conrey [5], and Young [15] among many other papers). Further, in the context of Dirichlet $L$-functions, we can enlarge the family by averaging also over the moduli $q$. Indeed, it is not difficult to use the large sieve to prove that

$$
\sum_{q \leq Q} \sum_{\chi \bmod q}^{\star}|L(1 / 2, \chi)|^{2}|A(1 / 2, \chi)|^{2} \ll Q^{2+\epsilon}
$$

where the sum is over primitive characters $\chi$, and

$$
A(1 / 2, \chi)=\sum_{n \leq Q^{\vartheta}} \lambda_{n} \frac{\chi(n)}{\sqrt{n}}
$$

is a Dirichlet polynomial with $\lambda_{n} \ll n^{\epsilon}$ and $\vartheta<1$. This gives an upper bound of (roughly) the right order of magnitude, while (as in conjecture (2)) we seek an asymptotic formula. In this paper, we use the asymptotic large sieve, developed by the authors in [8, 7, 6], to establish a $q$-analogue of the Balasubramanian, Conrey, and Heath-Brown conjecture when averaged also over $q$.

We now describe more precisely our main result. Let $\chi \bmod q$ be an even primitive character, and

$$
L(s, \chi)=\sum_{n=1}^{\infty} \chi(n) n^{-s}
$$

denote the corresponding Dirichlet $L$-function. The completed $L$-function

$$
\Lambda\left(\frac{1}{2}+s, \chi\right)=\left(\frac{q}{\pi}\right)^{\frac{s}{2}} \Gamma\left(\frac{1}{4}+\frac{s}{2}\right) L\left(\frac{1}{2}+s, \chi\right),
$$

satisfies the functional equation (with $|\epsilon(\chi)|=1$ )

$$
\Lambda\left(\frac{1}{2}+s\right)=\epsilon(\chi) \Lambda\left(\frac{1}{2}-s, \bar{\chi}\right)
$$

Let $W$ denote a fixed $C^{\infty}$ function, compactly supported on $[1,2]$, and let $\alpha$ and $\beta$ be "shifts." Our goal is to evaluate

$$
\Delta_{\alpha, \beta}(h, k ; Q):=\sum_{q} W\left(\frac{q}{Q}\right) \sum_{\chi \bmod q}^{b} \Lambda\left(\frac{1}{2}+\alpha, \chi\right) \Lambda\left(\frac{1}{2}+\beta, \bar{\chi}\right) \chi(h) \bar{\chi}(k),
$$


where the $b$ indicates that the sum is restricted to even primitive characters. The restriction to even characters is purely for convenience, so that the $\Gamma$-factors in the functional equation have the same shape, and one can consider in the same way odd primitive characters. Our interest is in the situation where the shifts $\alpha$ and $\beta$ are small, (precisely, $\alpha, \beta \ll 1 / \log Q$ ) but it may be possible to relax this and allow the shifts to be large (see [1]).

Theorem 1. Let $Q$ be large, and suppose the shifts $\alpha$ and $\beta$ are $\ll 1 / \log Q$. Then

$$
\begin{aligned}
\Delta_{\alpha, \beta}(h, k) & =\sum_{(q, h k)=1} W\left(\frac{q}{Q}\right)\left(\sum_{\chi \bmod q}^{b} 1\right)\left(\left(\frac{q}{\pi}\right)^{\frac{\alpha+\beta}{2}} \Gamma\left(\frac{1}{4}+\frac{\alpha}{2}\right) \Gamma\left(\frac{1}{4}+\frac{\beta}{2}\right) \frac{(h, k)^{1+\alpha+\beta}}{h^{\frac{1}{2}+\beta} k^{\frac{1}{2}+\alpha}} \zeta_{q}(1+\alpha+\beta)\right. \\
& \left.+\left(\frac{q}{\pi}\right)^{\frac{-\alpha-\beta}{2}} \Gamma\left(\frac{1}{4}-\frac{\alpha}{2}\right) \Gamma\left(\frac{1}{4}-\frac{\beta}{2}\right) \frac{(h, k)^{1-\alpha-\beta}}{h^{\frac{1}{2}-\alpha} k^{\frac{1}{2}-\beta}} \zeta_{q}(1-\alpha-\beta)\right)+\mathcal{E}_{h, k},
\end{aligned}
$$

where

$$
\zeta_{q}(s)=\zeta(s) \prod_{p \mid q}\left(1-\frac{1}{p^{s}}\right)
$$

and the remainder terms $\mathcal{E}_{h, k}$ satisfy

$$
\sum_{h, k \leq Q^{\vartheta}} \frac{\lambda_{h} \overline{\lambda_{k}}}{\sqrt{h k}} \mathcal{E}_{h, k}=O\left(Q^{2-(1-\vartheta) / 2+\epsilon}\right)
$$

uniformly for arbitrary complex numbers $\lambda_{h}$ with $\lambda_{h} \ll h^{\epsilon}$, and $\vartheta<1$.

In the statement of the theorem, and throughout the paper, $\epsilon$ will stand for an positive number that may be taken arbitrarily small, and its value may change from line to line. Thus, in the remainder terms in Theorem 1 we have obtained a power saving whenever $\vartheta$ is fixed below 1. In the situation $\alpha=\beta=0$ (as in the original formulation of the Balasubramanian, Conrey, and Heath-Brown conjecture), a little calculation shows that

$$
\sum_{q} W\left(\frac{q}{Q}\right) \sum_{\chi \bmod q}^{b}\left|L\left(\frac{1}{2}, \chi\right)\right|^{2} \chi(h) \bar{\chi}(k)
$$

has for its main term

$$
\sum_{(q, h k)=1} W\left(\frac{q}{Q}\right)\left(\sum_{\chi \bmod q}^{b} 1\right) \frac{\phi(q)}{q} \frac{(h, k)}{\sqrt{h k}}\left(\log \frac{q(h, k)^{2}}{\pi h k}+2 \gamma+\frac{\Gamma^{\prime}}{\Gamma}\left(\frac{1}{4}\right)+2 \sum_{p \mid q} \frac{\log p}{p-1}\right),
$$

with the error terms being controlled on average as in Theorem 1 .

In the main theorem, we have kept the main term in a natural form, which also suggests that such an asymptotic formula holds for each individual $q$ and not just on average over $q$. But one can readily give an asymptotic version of the main terms in Theorem 1 where the sum over $q$ has been executed. To state this cleanly, we need a little more notation. For brevity, we shall write

$$
W_{\alpha, \beta}(x)=x^{1+\frac{\alpha+\beta}{2}} W(x)
$$


and write its Mellin transform as

$$
\widetilde{W}_{\alpha, \beta}(s)=\int_{0}^{\infty} W_{\alpha, \beta}(x) x^{s} \frac{d x}{x} .
$$

For any complex number $s$, put

$$
\Phi(q, s)=\prod_{p \mid q}\left(1-\frac{1}{q^{s}}\right)
$$

and finally, define for complex numbers $s$ and $w$ with $\operatorname{Re}(w)>0$ and $\operatorname{Re}(s+w)>1$

$$
\mathcal{P}(q ; w, s)=\prod_{p \nmid q}\left(1-\frac{1}{p^{s+w}}-\frac{2}{p^{1+w}}+\frac{2}{p^{1+s+w}}+\frac{1}{p^{2+2 w}}-\frac{1}{p^{2+2 w+s}}\right) .
$$

Then a small calculation allows us to recast the main term in Theorem 1 as

$$
\begin{aligned}
\frac{Q^{2}}{2} \Phi(h k, 1)\left(\widetilde{W}_{\alpha, \beta}(1)\left(\frac{Q}{\pi}\right)^{\frac{\alpha+\beta}{2}} \Gamma\left(\frac{1}{4}+\frac{\alpha}{2}\right) \Gamma\left(\frac{1}{4}+\frac{\beta}{2}\right) \frac{(h, k)^{1+\alpha+\beta}}{h^{1 / 2+\beta} k^{1 / 2+\alpha}}\right. \\
\times \zeta(1+\alpha+\beta) \mathcal{P}(h k ; 1,1+\alpha+\beta) \\
+\widetilde{W}_{-\beta,-\alpha}(1)\left(\frac{Q}{\pi}\right)^{\frac{-\alpha-\beta}{2}} \Gamma\left(\frac{1}{4}-\frac{\alpha}{2}\right) \Gamma\left(\frac{1}{4}-\frac{\beta}{2}\right) \frac{(h, k)^{1-\beta-\alpha}}{h^{1 / 2-\alpha} k^{1 / 2-\beta}} \\
\times \zeta(1-\alpha-\beta) \mathcal{P}(h k ; 1,1-\beta-\alpha))
\end{aligned}
$$

and in fact it is in this form that we will establish Theorem 1 .

As mentioned earlier, Theorem 1 establishes a $q$-analogue of the conjecture of Balasubramanian, Conrey, and Heath-Brown, on average over $q$. The extra average over $q$ means that one does not obtain improvements to the convexity bound for Dirichlet $L$-functions from Theorem 1. However, one can use Theorem 1 to obtain a modest improvement to the result of [7] on critical zeros of Dirichlet $L$-function. More precisely, let $N(T, \chi)$ be the number of zeros of $L(s, \chi)$ whose real parts are positive and imaginary parts at most $T$, and let $N_{0}(T, \chi)$ be the number of such zeros that are on the critical line. Then for $Q$ sufficiently large, and $(\log Q)^{6} \leq T \leq(\log Q)^{A}$ (for any $\left.A>6\right)$ we have

$$
\sum_{q} W\left(\frac{q}{Q}\right) \sum_{\chi \bmod q}^{b} N_{0}(T, \chi)>\frac{3}{5} \sum_{q} W\left(\frac{q}{Q}\right) \sum_{\chi \bmod q}^{b} N(T, \chi) .
$$

The earlier result in [7] gave 57\% instead of $60 \%$ above, and the additional saving comes from the flexibility of allowing arbitrary coefficients in the mollifier, which permits the choice of mollifier introduced by Feng [9]. We also note one difference from our earlier work on the asymptotic large sieve in the context of moments of Dirichlet $L$-function [8]. Namely, the earlier work required also a small average in $t$-aspect, whereas in the context of Theorem 1 we are able to treat central values of $L$-functions without this extra $t$-averaging. 


\section{Preliminary CONSIDERAtions}

Throughout, we shall assume that the shifts $\alpha$ and $\beta$ are non-zero, and $\alpha \neq \pm \beta$; the final answers will be uniformly continuous in $\alpha$ and $\beta$, so that the results will hold in the edge cases. We begin with an "approximate functional equation" for the $L$-functions in our average. Define

$$
\widetilde{V}_{\alpha, \beta}(s)=\Gamma\left(\frac{s+1 / 2+\alpha}{2}\right) \Gamma\left(\frac{s+1 / 2+\beta}{2}\right)\left(1-\left(\frac{2 s}{\alpha+\beta}\right)^{2}\right),
$$

which vanishes at $s= \pm \frac{1}{2}(\alpha+\beta)$ simplifying some later calculations. For any positive real number $x$, put

$$
V_{\alpha, \beta}(x)=\frac{1}{2 \pi i} \int_{(1)} \widetilde{V}_{\alpha, \beta}(s) x^{-s} \frac{d s}{s} .
$$

The function $V_{\alpha, \beta}(x)$, which is a variant of the Mellin transform, essentially picks out values of $x$ that are of bounded size. For large $x$, by moving the line of integration in (12) to the right we may establish that $V_{\alpha, \beta}(x) \ll \exp (-\tau x)$ for a suitable positive constant $\tau$. For small $x$, by moving the line of integration in $(12)$ to $\operatorname{Re}(s)=-\frac{1}{2}+\epsilon$ we can establish that

$$
V_{\alpha, \beta}(x)=\Gamma\left(\frac{1}{4}+\frac{\alpha}{2}\right) \Gamma\left(\frac{1}{4}+\frac{\beta}{2}\right)+O\left(x^{\frac{1}{2}-\epsilon}\right) .
$$

We shall use these, and related facts about $V_{\alpha, \beta}(x)$ without further comment below.

Lemma 1. With notations as above

$$
\Lambda\left(\frac{1}{2}+\alpha, \chi\right) \Lambda\left(\frac{1}{2}+\beta, \bar{\chi}\right)=S(\alpha, \beta ; \chi)+S(-\beta,-\alpha ; \chi)
$$

where

$$
S(\alpha, \beta ; \chi)=\left(\frac{q}{\pi}\right)^{\frac{\alpha+\beta}{2}} \sum_{m, n} \frac{\chi(m) \overline{\chi(n)}}{m^{1 / 2+\alpha} n^{1 / 2+\beta}} V_{\alpha, \beta}\left(\frac{\pi m n}{q}\right) .
$$

Proof. We sketch quickly the standard proof. We start with

$$
\frac{1}{2 \pi i} \int_{(1)} \Lambda\left(\frac{1}{2}+s+\alpha, \chi\right) \Lambda\left(\frac{1}{2}+s+\beta, \bar{\chi}\right)\left(1-\left(\frac{2 s}{\alpha+\beta}\right)^{2}\right) \frac{d s}{s} .
$$

Since we are in the region of absolute convergence, expanding out $L\left(\frac{1}{2}+s+\alpha, \chi\right) L\left(\frac{1}{2}+s+\beta, \bar{\chi}\right)$ into its Dirichlet series, and integrating term by term, this equals $S(\alpha, \beta ; \chi)$. On the other hand, we may move the line of integration to $\operatorname{Re}(s)=-1$. We encounter a pole at $s=0$ which leaves the residue $\Lambda\left(\frac{1}{2}+\alpha, \chi\right) \Lambda\left(\frac{1}{2}+\beta, \bar{\chi}\right)$. Thus the quantity in (14) also equals

$$
\Lambda\left(\frac{1}{2}+\alpha, \chi\right) \Lambda\left(\frac{1}{2}+\beta, \bar{\chi}\right)+\frac{1}{2 \pi i} \int_{(-1)} \Lambda\left(\frac{1}{2}+s+\alpha, \chi\right) \Lambda\left(\frac{1}{2}+s+\beta, \bar{\chi}\right)\left(1-\left(\frac{2 s}{\alpha+\beta}\right)^{2}\right) \frac{d s}{s} .
$$

Using the functional equation and replacing $s$ by $-s$, we see that the integral above equals $-S(-\beta,-\alpha ; \chi)$, which completes the proof of the lemma. 
The next lemma follows from the orthogonality relations for characters, adapted to handle even primitive characters.

Lemma 2. If $(m n, q)=1$, then

$$
\sum_{\chi \bmod q}^{b} \chi(m) \overline{\chi(n)}=\frac{1}{2}\left(\sum_{\substack{d|q \\ d|(m-n)}} \phi(d) \mu\left(\frac{q}{d}\right)+\sum_{\substack{d|q \\ d|(m+n)}} \phi(d) \mu\left(\frac{q}{d}\right)\right) .
$$

It will be convenient to adopt the notation $d \mid(m \pm n)$ to indicate either $d \mid(m+n)$ or $d \mid(m-$ $n)$, and if both possibilities occur then the variable $d$ is counted twice. Thus, for example, we may combine the two sums appearing in Lemma 2 and write $\sum_{d|q, d|(m \pm n)} \phi(d) \mu(q / d)$.

Applying Lemmas 1 and 2 we obtain

$$
\Delta_{\alpha, \beta}(h, k)=T_{\alpha, \beta}(h, k)+T_{-\beta,-\alpha}(h, k)
$$

where, writing $q=c d$, and recalling the notation (6),

$$
T_{\alpha, \beta}(h, k)=\frac{Q}{2}\left(\frac{Q}{\pi}\right)^{\frac{\alpha+\beta}{2}} \sum_{m, n} \sum_{\substack{(c d, m n h k)=1 \\ d \mid m h \pm n k}} \frac{\mu(c) \phi(d)}{c d} \frac{1}{m^{\frac{1}{2}+\alpha} n^{\frac{1}{2}+\beta}} V_{\alpha, \beta}\left(\frac{\pi m n}{c d}\right) W_{\alpha, \beta}\left(\frac{c d}{Q}\right) .
$$

Our goal is now to evaluate $T_{\alpha, \beta}(h, k)$. To this end, we introduce a parameter $C$ (which will eventually be chosen as a small power of $Q)$ and split $T_{\alpha, \beta}(h, k)$ as

$$
T_{\alpha, \beta}(h, k)=\mathcal{L}_{\alpha, \beta}(h, k)+\mathcal{D}_{\alpha, \beta}(h, k)+\mathcal{U}_{\alpha, \beta}(h, k),
$$

depending on whether $c<C$, and whether $m h=n k$. Precisely, we write

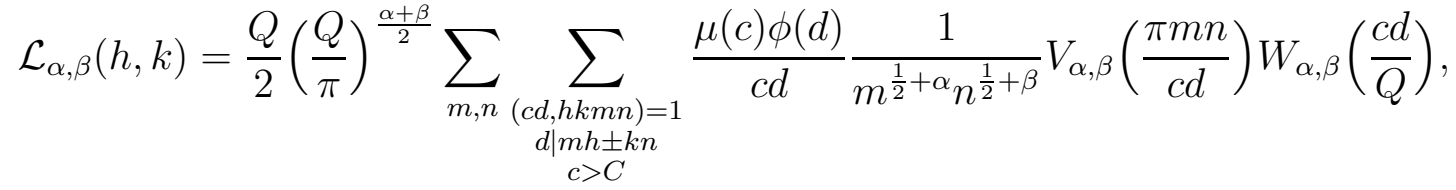

$$
\begin{aligned}
& \mathcal{D}_{\alpha, \beta}(h, k)=\frac{Q}{2}\left(\frac{Q}{\pi}\right)^{\frac{\alpha+\beta}{2}} \sum_{\substack{m, n \\
m h=n k}} \sum_{\substack{(c d, h k m n)=1 \\
c \leq C}} \frac{\mu(c) \phi(d)}{c d} \frac{1}{m^{\frac{1}{2}+\alpha} n^{\frac{1}{2}+\beta}} V_{\alpha, \beta}\left(\frac{\pi m n}{c d}\right) W_{\alpha, \beta}\left(\frac{c d}{Q}\right),
\end{aligned}
$$

and

$$
\mathcal{U}_{\alpha, \beta}(h, k)=\frac{Q}{2}\left(\frac{Q}{\pi}\right)^{\frac{\alpha+\beta}{2}} \sum_{\substack{m, n \\ m h \neq n k}} \sum_{\substack{c d, h k m n)=1 \\ d \mid m h \pm k n \\ c \leq C}} \frac{\mu(c) \phi(d)}{c d} \frac{1}{m^{\frac{1}{2}+\alpha} n^{\frac{1}{2}+\beta}} V_{\alpha, \beta}\left(\frac{\pi m n}{c d}\right) W_{\alpha, \beta}\left(\frac{c d}{Q}\right) .
$$

In Section 3 we shall give an expression for the diagonal terms $\mathcal{D}_{\alpha, \beta}(h, k)$; when $\vartheta<1 / 2$ these terms account for the entire main term contribution in the main theorem, but for $\vartheta>1 / 2$ there are additional main terms arising from the off diagonal terms. In Section 4 we treat the terms $\mathcal{L}_{\alpha, \beta}(h, k)$, isolating a potentially large main term. The most difficult part of our analysis involves the terms $\mathcal{U}_{\alpha, \beta}(h, k)$, and this is carried out in Sections 5, 6, and 7. Briefly, we must identify another large term which cancels precisely the corresponding 
contribution in $\mathcal{L}_{\alpha, \beta}(h, k)$, and then we are left with a new main term which when combined with the diagonal term $\mathcal{D}_{-\beta,-\alpha}(h, k)$ leads finally to the main term of the theorem.

\section{The Diagonal Terms $\mathcal{D}_{\alpha, \beta}(h, k)$}

This section gives a preliminary treatment of the diagonal terms $\mathcal{D}_{\alpha, \beta}(h, k)$, deriving a formula for this term which will be useful in conjunction with similar formulae arising from other main terms (yet to be identified). If we write $h=H(h, k)$ and $k=K(h, k)$, so that $(H, K)=1$, and the relation $m h=n k$ implies that $m=K \ell$ and $n=H \ell$ for some natural number $\ell$. Therefore

$$
\mathcal{D}_{\alpha, \beta}(h, k)=\frac{Q}{2}\left(\frac{Q}{\pi}\right)^{\frac{\alpha+\beta}{2}} \sum_{\ell} \sum_{\substack{(c d, \ell h k)=1 \\ c \leq C}} \frac{\mu(c) \phi(d)}{c d} \frac{1}{\ell^{1+\alpha+\beta} H^{\frac{1}{2}+\beta} K^{\frac{1}{2}+\alpha}} V_{\alpha, \beta}\left(\frac{\pi \ell^{2} H K}{c d}\right) W_{\alpha, \beta}\left(\frac{c d}{Q}\right) .
$$

We can extend the sum in $c$ to include all natural numbers $c$, incurring an acceptable error term of size (using that $W$ is supported on $[1,2]$ and that $V_{\alpha, \beta}(x)$ is exponentially small for large $x$ )

$$
\ll \frac{Q}{(H K)^{\frac{1}{2}}}(\log Q) \sum_{c>C} \frac{1}{c} \sum_{d \leq 2 Q / c} \frac{\phi(d)}{d} \ll \frac{Q^{2}(\log Q)}{(H K)^{\frac{1}{2}} C} .
$$

If we sum this error term over all $h$ and $k$, we obtain

$$
\sum_{h, k \leq Q^{\vartheta}} \frac{\left|\lambda_{h} \lambda_{k}\right|}{\sqrt{h k}} \frac{Q^{2} \log Q}{\sqrt{H K} C} \ll \frac{Q^{2+\epsilon}}{C} .
$$

Since this is under control (we shall eventually choose $C=Q^{(1-\vartheta) / 2}$ ), we shall omit this error term in our further discussion of $\mathcal{D}_{\alpha, \beta}(h, k)$.

Write

$$
\phi^{*}(q)=\sum_{c d=q} \mu(c) \phi(d)
$$

which counts the number of primitive characters mod $q$. By (19) and (20), and grouping together terms with $c d=q$, we have (omitting the error term as mentioned above)

$$
\mathcal{D}_{\alpha, \beta}(h, k)=\frac{Q(Q / \pi)^{\frac{\alpha+\beta}{2}}}{2 H^{\frac{1}{2}+\beta} K^{\frac{1}{2}+\alpha}} \sum_{\ell} \sum_{(q, \ell h k)=1} \frac{\phi^{*}(q)}{q} \frac{1}{\ell^{1+\alpha+\beta}} V_{\alpha, \beta}\left(\frac{\pi \ell^{2} H K}{q}\right) W_{\alpha, \beta}\left(\frac{q}{Q}\right) .
$$

Recalling the definition of $V_{\alpha, \beta}(x)$ (see (11) and (12)), we may express the sum over $\ell$ in the main term above as (for any $\epsilon>0$, and keeping in mind that $\alpha$ and $\beta$ are $\ll 1 / \log Q$ )

$$
\frac{1}{2 \pi i} \int_{(\epsilon)} \widetilde{V}_{\alpha, \beta}(s)\left(\frac{q}{\pi H K}\right)^{s} \zeta_{q}(1+2 s+\alpha+\beta) \frac{d s}{s},
$$


where

$$
\zeta_{q}(s)=\prod_{p \nmid q}\left(1-\frac{1}{p^{s}}\right)^{-1}=\zeta(s) \Phi(q, s), \quad \text { with } \quad \Phi(q, s)=\prod_{p \mid q}\left(1-\frac{1}{p^{s}}\right) .
$$

To proceed further, and evaluate the sum over $q$ in (22), the following lemma will be useful.

Lemma 3. Suppose $s$ and $w$ are complex numbers with $\operatorname{Re}(s)$ and $\operatorname{Re}(w)$ larger than 1 . Then

$$
\sum_{(q, h k)=1} \frac{\phi^{*}(q)}{q^{1+w}} \zeta_{q}(s)=\zeta(w) \zeta(s) \Phi(h k, w) \mathcal{P}(h k ; w, s)
$$

where $\mathcal{P}(h k ; w, s)$ was defined in (9)).

Proof. Both sides of the claimed identity can be expressed as products over primes, and so it is enough to check that the Euler factors match up. For a prime $p \mid h k$ the Euler factor on both sides is simply $\left(1-p^{-s}\right)^{-1}$. If $p \nmid h k$, the Euler factor on the left side is

$$
\left(1-\frac{1}{p^{s}}\right)^{-1}+\frac{(p-2)}{p^{1+w}}+\sum_{j=2}^{\infty}\left(1-\frac{1}{p}\right)^{2} p^{-w j}=\left(1-\frac{1}{p^{s}}\right)^{-1}+\frac{(p-2)}{p^{1+w}}+\left(1-\frac{1}{p}\right)^{2}\left(1-\frac{1}{p^{w}}\right)^{-1} \frac{1}{p^{2 w}} .
$$

Multiply this by $\left(1-p^{-w}\right)\left(1-p^{-s}\right)$, and then a small calculation shows that the result matches the Euler factor appearing in (9).

Now we return to our analysis of $\mathcal{D}_{\alpha, \beta}(h, k)$, recalling that its main term in (22) equals

$$
\frac{Q(Q / \pi)^{\frac{\alpha+\beta}{2}}}{2 H^{\frac{1}{2}+\beta} K^{\frac{1}{2}+\alpha}} \frac{1}{2 \pi i} \int_{(\epsilon)} \frac{\widetilde{V}_{\alpha, \beta}(s)}{(\pi H K)^{s}} \sum_{(q, h k)=1} W_{\alpha, \beta}\left(\frac{q}{Q}\right) \frac{\phi^{*}(q)}{q} q^{s} \zeta_{q}(1+2 s+\alpha+\beta) \frac{d s}{s} .
$$

By Mellin inversion, we may write

$$
W_{\alpha, \beta}(x)=\frac{1}{2 \pi i} \int_{(c)} \widetilde{W}_{\alpha, \beta}(w) x^{-w} d w, \quad \text { where } \quad \widetilde{W}_{\alpha, \beta}(w)=\int_{0}^{\infty} x^{w} W_{\alpha, \beta}(x) \frac{d x}{x} .
$$

Therefore the sum over $q$ in (24) becomes (for suitably large $c$ )

$$
\frac{1}{2 \pi i} \int_{(c)} \widetilde{W}_{\alpha, \beta}(w) Q^{w} \sum_{(q, h k)=1} \frac{\phi^{*}(q)}{q} q^{-w+s} \zeta_{q}(1+2 s+\alpha+\beta) d w
$$

which is, by Lemma 3 ,

$$
\frac{1}{2 \pi i} \int_{(c)} \widetilde{W}_{\alpha, \beta}(w) Q^{w} \zeta(w-s) \zeta(1+2 s+\alpha+\beta) \Phi(h k, w-s) \mathcal{P}(h k ; w-s, 1+2 s+\alpha+\beta) d w .
$$

We move the line of integration to $\operatorname{Re}(w)=\epsilon$, passing a pole at $w=1+s$. Thus the integral above equals

$$
\widetilde{W}_{\alpha, \beta}(1+s) Q^{1+s} \zeta(1+2 s+\alpha+\beta) \Phi(h k, 1) \mathcal{P}(h k ; 1,1+2 s+\alpha+\beta)+O\left(Q^{\epsilon}\right) .
$$


Using the above analysis in (24) and (22), we conclude that

$$
\begin{aligned}
\mathcal{D}_{\alpha, \beta}(h, k) & =O\left(\frac{Q^{2}(\log Q)^{2}}{C \sqrt{H K}}+\frac{Q^{1+\epsilon}}{\sqrt{H K}}\right)+\left(\frac{Q}{\pi}\right)^{\frac{\alpha+\beta}{2}} \frac{Q^{2} \Phi(h k, 1)}{2 H^{\frac{1}{2}+\beta} K^{\frac{1}{2}+\alpha}} \frac{1}{2 \pi i} \\
(26) & \times \int_{(\epsilon)} \widetilde{V}_{\alpha, \beta}(s) \widetilde{W}_{\alpha, \beta}(1+s)\left(\frac{Q}{\pi H K}\right)^{s} \zeta(1+2 s+\alpha+\beta) \mathcal{P}(h k ; 1,1+2 s+\alpha+\beta) \frac{d s}{s} .
\end{aligned}
$$

We shall return to this expression later, combining it with other main terms that will arise.

\section{The TERMS $\mathcal{L}_{\alpha, \beta}(h, k)$}

To treat $\mathcal{L}_{\alpha, \beta}$, we replace the condition $d \mid(m h \pm n k)$ by a sum over all even characters modulo $d$. Thus

$$
\begin{aligned}
\mathcal{L}_{\alpha, \beta}(h, k) & =Q\left(\frac{Q}{\pi}\right)^{\frac{\alpha+\beta}{2}} \sum_{m, n} \sum_{\substack{c, d \\
c>C \\
(c d, h k m n)=1}} \sum_{\substack{\psi \text { mod } d \\
\psi \text { even }}} \frac{\mu(c)}{c d} \frac{\psi(m h) \bar{\psi}(n k)}{m^{\frac{1}{2}+\alpha} n^{\frac{1}{2}+\beta}} V_{\alpha, \beta}\left(\frac{\pi m n}{c d}\right) W_{\alpha, \beta}\left(\frac{c d}{Q}\right) \\
& =\mathcal{L}_{\alpha, \beta}^{0}(h, k)+\mathcal{L}_{\alpha, \beta}^{r}(h, k),
\end{aligned}
$$

where $\mathcal{L}_{\alpha, \beta}^{0}(h, k)$ denotes the contribution of the principal character $\psi=\psi_{0}$, and $\mathcal{L}_{\alpha, \beta}^{r}(h, k)$ denotes the contribution from the remaining non-principal characters $\psi$. It may appear that we are going backwards in this step by introducing characters yet again, but the advantage is that the conductor of these characters (essentially $d$ ) is now smaller (at most $Q / C$ ) which allows us to use the large sieve inequality efficiently.

We first simplify a little the main term contribution of $\mathcal{L}_{\alpha, \beta}^{0}(h, k)$, and then estimate the contribution of the remainder terms $\mathcal{L}_{\alpha, \beta}^{r}(h, k)$. From its definition, we have

$$
\mathcal{L}_{\alpha, \beta}^{0}(h, k)=Q\left(\frac{Q}{\pi}\right)^{\frac{\alpha+\beta}{2}} \sum_{m, n} \sum_{\substack{c, d \\ c>C \\(c d, h k m n)=1}} \frac{\mu(c)}{c d} \frac{1}{m^{\frac{1}{2}+\alpha} n^{\frac{1}{2}+\beta}} V_{\alpha, \beta}\left(\frac{\pi m n}{c d}\right) W_{\alpha, \beta}\left(\frac{c d}{Q}\right) .
$$

Group terms according to $c d=q$ and since $q$ is of size $Q$ and so in particular $q>1$, note that

$$
\sum_{\substack{c d=q \\ c>C}} \mu(c)=-\sum_{\substack{c d=q \\ c \leq C}} \mu(c) .
$$

Thus we may rewrite the sum over $c$ and $d$ in (28) as

$$
-\sum_{\substack{c \leq C \\(c, h k m n)=1}} \sum_{(d, h k m n)=1} \frac{\mu(c)}{c d} V_{\alpha, \beta}\left(\frac{\pi m n}{c d}\right) W_{\alpha, \beta}\left(\frac{c d}{Q}\right) .
$$


Since $\sum_{d \leq x,(d, h k m n)=1} 1=x \phi(h k m n) / h k m n+O\left((h k m n)^{\epsilon}\right)$, using partial summation the above equals

$$
-\sum_{\substack{c \leq C \\(c, h k m n)=1}} \frac{\mu(c)}{c} \frac{\phi(h k m n)}{h k m n} \int_{0}^{\infty} V_{\alpha, \beta}\left(\frac{\pi m n}{c x}\right) W_{\alpha, \beta}\left(\frac{c x}{Q}\right) \frac{d x}{x}+O\left(\frac{C}{Q}(h k m n)^{\epsilon} e^{-\tau m n / Q}\right),
$$

for some positive constant $\tau$. Inserting this in (28), we conclude that

$$
\begin{aligned}
& \mathcal{L}_{\alpha, \beta}^{0}(h, k)=O\left(Q^{\frac{1}{2}+\epsilon} C\right) \\
& (29) \quad-Q\left(\frac{Q}{\pi}\right)^{\frac{\alpha+\beta}{2}} \sum_{m, n} \frac{1}{m^{\frac{1}{2}+\alpha}} \frac{1}{n^{\frac{1}{2}+\beta}} \frac{\phi(m n h k)}{m n h k} \sum_{\substack{(c, h k m n)=1 \\
c \leq C}} \frac{\mu(c)}{c} \int_{0}^{\infty} V_{\alpha, \beta}\left(\frac{\pi m n}{Q x}\right) W_{\alpha, \beta}(x) \frac{d x}{x} .
\end{aligned}
$$

We stop our treatment of $\mathcal{L}_{\alpha, \beta}^{0}(h, k)$ here; it will turn out that this expression cancels another main term arising from our treatment of $\mathcal{U}_{\alpha, \beta}(h, k)$.

The next lemma gives a satisfactory bound for $\mathcal{L}_{\alpha, \beta}^{r}(h, k)$, on average over $h$ and $k$.

Lemma 4. Suppose $\lambda_{h} \ll h^{\epsilon}$ for $1 \leq h \leq Q^{\vartheta}$. Then

$$
\sum_{h, k \leq Q^{\vartheta}} \frac{\lambda_{h} \overline{\lambda_{k}}}{\sqrt{h k}} \mathcal{L}_{\alpha, \beta}^{r}(h, k) \ll \frac{Q^{2+\epsilon}}{C}+Q^{1+\vartheta+\epsilon} .
$$

Proof. Using the Mellin transform (12) in the definition of $\mathcal{L}_{\alpha, \beta}^{r}(h, k)$, we obtain

$$
\begin{aligned}
\mathcal{L}_{\alpha, \beta}^{r}(h, k)=Q\left(\frac{Q}{\pi}\right)^{\frac{\alpha+\beta}{2}} & \sum_{\substack{c, d \\
c>C \\
(c d, h k)=1}} \sum_{\substack{\psi \bmod d \\
\text { even } \\
\psi \neq \psi_{0}}} \frac{\mu(c)}{c d} W_{\alpha, \beta}\left(\frac{c d}{Q}\right) \psi(h) \overline{\psi(k)} \\
& \times \frac{1}{2 \pi i} \int_{\left(\frac{1}{2}+\epsilon\right)} \widetilde{V}_{\alpha, \beta}(s)\left(\frac{c d}{\pi}\right)^{s} \sum_{\substack{m, n \\
(c d, m n)=1}} \frac{\psi(m) \overline{\psi(n)}}{m^{\frac{1}{2}+\alpha+s} n^{\frac{1}{2}+\beta+s}} \frac{d s}{s} .
\end{aligned}
$$

The sum over $m$ and $n$ may be written as

$$
L\left(\frac{1}{2}+\alpha+s, \psi\right) L\left(\frac{1}{2}+\beta+s, \bar{\psi}\right) \prod_{p \mid c}\left(1-\frac{\psi(p)}{p^{\frac{1}{2}+\alpha+s}}\right)\left(1-\frac{\overline{\psi(p)}}{p^{\frac{1}{2}+\beta+s}}\right)
$$

and since $\psi \neq \psi_{0}$, we may move the line of integration to $\operatorname{Re}(s)=\epsilon$ without encountering any poles. If $s=\epsilon+i t$ then $\widetilde{V}_{\alpha, \beta}(s) / s$ is $\ll e^{-\tau|t|}$ for some positive constant $\tau$, and further 
the product over $p \mid c$ in (30) may be bounded by $\ll c^{\epsilon}$. Therefore, we find (with $s=\epsilon+i t$ )

$$
\begin{aligned}
\sum_{h, k \leq Q^{\vartheta}} \frac{\lambda_{h} \overline{\lambda_{k}}}{\sqrt{h k}} \mathcal{L}_{\alpha, \beta}^{r}(h, k) \ll Q^{\epsilon} \sum_{\substack{c, d \\
c>C}} W\left(\frac{c d}{Q}\right) \sum_{\substack{\psi \text { mod } d \\
\psi \text { even } \\
\psi \neq \psi_{0}}}\left|\sum_{\substack{h \leq Q^{\vartheta} \\
(h, c)=1}} \frac{\lambda_{h} \psi(h)}{\sqrt{h}}\right|^{2} \\
\quad \times \int_{-\infty}^{\infty} e^{-\tau|t|}\left|L\left(\frac{1}{2}+\alpha+s, \psi\right) L\left(\frac{1}{2}+\beta+s, \bar{\psi}\right)\right| d t .
\end{aligned}
$$

Given $c>C$, consider the sum over $d$ above ( $\operatorname{so} d$ is of size $Q / c$ ). We regroup the characters $\psi$ above based on primitive characters; thus suppose $d=r u$ and that $\psi$ is induced by a primitive character $\bmod u$. Therefore the sum over $d$ above may be bounded by

$$
\begin{aligned}
Q^{\epsilon} \sum_{r \ll Q / c} \sum_{u \ll Q /(c r)} & \sum_{\substack{\psi \bmod u \\
\text { even, primitive }}}\left|\sum_{\substack{h \leq Q^{\vartheta} \\
(h, c r)=1}} \frac{\lambda_{h} \psi(h)}{\sqrt{h}}\right|^{2} \\
& \times \int_{-\infty}^{\infty} e^{-\tau|t|}\left(\left|L\left(\frac{1}{2}+\alpha+s, \psi\right)\right|^{2}+\left|L\left(\frac{1}{2}+\beta+s, \psi\right)\right|^{2}\right) d t .
\end{aligned}
$$

Now applications of the large sieve show that

$$
\sum_{\substack{\psi \bmod u \\ \psi \text { primitive }}}\left(\left|L\left(\frac{1}{2}+\alpha+s, \psi\right)\right|^{4}+\left|L\left(\frac{1}{2}+\beta+s, \psi\right)\right|^{4}\right) \ll u^{1+\epsilon}|s|^{A},
$$

for some constant $A$, and that

$$
\sum_{u \ll Q /(c r)} \sum_{\substack{\psi \bmod u \\ \psi \text { primitive }}}\left|\sum_{\substack{h \leq Q^{\vartheta} \\(h, c r)=1}} \frac{\lambda_{h} \psi(h)}{\sqrt{h}}\right|^{4} \ll Q^{\epsilon}\left(\frac{Q^{2}}{c^{2} r^{2}}+Q^{2 \vartheta}\right) .
$$

Using these estimates together with Cauchy-Schwarz in (32), we see that the quantity in $(32)$ is

$$
\ll Q^{\epsilon} \sum_{r \ll Q / c} \frac{Q}{c r}\left(\frac{Q}{c r}+Q^{\vartheta}\right) \ll \frac{Q^{2+\epsilon}}{c^{2}}+\frac{Q^{1+\vartheta+\epsilon}}{c} .
$$

Inserting this estimate into (31) and summing over $c$ with $C \leq c \ll Q$, the lemma follows.

\section{The terms $\mathcal{U}_{\alpha, \beta}(h, k)$ : Switching to The COMPlementary MOdulus}

We begin our treatment of the terms $\mathcal{U}_{\alpha, \beta}(h, k)$, which forms the hardest part of our analysis. Recall, from (18), the definition

$$
\mathcal{U}_{\alpha, \beta}(h, k)=\frac{Q}{2}\left(\frac{Q}{\pi}\right)^{\frac{\alpha+\beta}{2}} \sum_{\substack{m, n \\ m h \neq n k}} \sum_{\substack{c d, h k m n)=1 \\ d \mid m h \pm k n \\ c \leq C}} \frac{\mu(c) \phi(d)}{c d} \frac{1}{m^{\frac{1}{2}+\alpha} n^{\frac{1}{2}+\beta}} V_{\alpha, \beta}\left(\frac{\pi m n}{c d}\right) W_{\alpha, \beta}\left(\frac{c d}{Q}\right)
$$


Our aim is to replace occurrences of $d$ (which is a divisor of $|m h \pm n k|)$ with its complementary divisor (essentially $|m h \pm n k| / d$ ) which will be of smaller size. The presence of the factor $\phi(d)$ and the coprimality condition $(d, h k m n)=1$, makes this a little complicated, entailing extra applications of Möbius inversion. Treating $m, n$ and $c$ as given (with $m h \neq k n)$, consider the sum over $d$ in (33). Write $(m h, n k)=g$, and then the conditions $d \mid(m h \pm n k)$ and $(d, h k m n)=1$ are equivalent to $d \mid(m h \pm n k) / g$ and $(d, g)=1$. Therefore, we are interested in

$$
\sum_{\substack{(d, g)=1 \\ d \mid(|m h \pm n k| / g)}} \frac{\phi(d)}{d} W_{\alpha, \beta}\left(\frac{c d}{Q}\right) V_{\alpha, \beta}\left(\frac{\pi m n}{c d}\right) .
$$

Writing $\phi(d) / d=\sum_{e f=d} \mu(e) / e$, the above becomes

$$
\sum_{(e, g)=1} \frac{\mu(e)}{e} \sum_{\substack{(f, g)=1 \\ e f \mid(|m h \pm n k| / g)}} W_{\alpha, \beta}\left(\frac{c e f}{Q}\right) V_{\alpha, \beta}\left(\frac{\pi m n}{c e f}\right)
$$

Lastly, we express the condition $(f, g)=1$ using Möbius inversion $\sum_{a|g, a| f} \mu(a)$; thus with $a b=f$, the sum above is

$$
\sum_{(e, g)=1} \sum_{a \mid g} \sum_{\substack{b \\ a b e \mid(|m h \pm n k| / g)}} \mu(a) \frac{\mu(e)}{e} W_{\alpha, \beta}\left(\frac{c e a b}{Q}\right) V_{\alpha, \beta}\left(\frac{\pi m n}{c e a b}\right) .
$$

We now introduce the complementary modulus $\ell$ via the relation $|m h \pm n k|=$ geab $\ell$, thereby converting the sum over $b$ into one over $\ell$. Thus the quantity in (34) may be recast as

$$
\sum_{\substack{(e, g)=1 \\ a \mid g}} \sum_{\substack{\ell \\ a e \ell \mid(|m h \pm n k| / g)}} \mu(a) \frac{\mu(e)}{e} W_{\alpha, \beta}\left(\frac{c|m h \pm n k|}{Q g \ell}\right) V_{\alpha, \beta}\left(\frac{\pi m n g \ell}{c|m h \pm n k|}\right) .
$$

Inserting this in (33), we conclude that

$$
\begin{gathered}
\mathcal{U}_{\alpha, \beta}(h, k)=\frac{Q}{2}\left(\frac{Q}{\pi}\right)^{\frac{\alpha+\beta}{2}} \sum_{\substack{(c, h k)=1 \\
c \leq C}} \frac{\mu(c)}{c} \sum_{\substack{m, n \\
(c, m n)=1 \\
m h \neq n k}} \sum_{\substack{a \mid g \\
(e, g)=1}} \mu(a) \frac{\mu(e)}{e} \frac{1}{m^{\frac{1}{2}+\alpha}} \frac{1}{n^{\frac{1}{2}+\beta}} \\
\sum_{\substack{\ell \\
a e \ell \mid(|m h \pm n k| / g)}} W_{\alpha, \beta}\left(\frac{c|m h \pm n k|}{Q g \ell}\right) V_{\alpha, \beta}\left(\frac{\pi m n g \ell}{c|m h \pm n k|}\right) .
\end{gathered}
$$

Since $(m h, n k)=g$, in (35) note that ael must be coprime to $m h / g$ and $n k / g$. Therefore the congruence $(m h \pm n k) / g \equiv 0 \bmod$ ael may be detected using the characters $\bmod$ ael. We isolate the contribution of the principal character $\bmod$ ael as $\mathcal{U}_{\alpha, \beta}^{0}(h, k)$ and denote by 
$\mathcal{U}_{\alpha, \beta}^{r}(h, k)$ the contribution of the remaining non-principal characters. Thus

$$
\begin{aligned}
\mathcal{U}_{\alpha, \beta}^{0}(h, k)=\frac{Q}{2}\left(\frac{Q}{\pi}\right)^{\frac{\alpha+\beta}{2}} & \sum_{\substack{(c, h k)=1 \\
c \leq C}} \frac{\mu(c)}{c} \sum_{\substack{m, n \\
(c, m n)=1 \\
m h \neq n k}} \sum_{\substack{a \mid g \\
(e, g)=1}} \mu(a) \frac{\mu(e)}{e} \frac{1}{m^{\frac{1}{2}+\alpha}} \frac{1}{n^{\frac{1}{2}+\beta}} \\
& \sum_{\substack{\ell \\
\left(a e \ell, m n h k / g^{2}\right)=1}} \frac{1}{\phi(a e \ell)} W_{\alpha, \beta}\left(\frac{c|m h \pm n k|}{Q g \ell}\right) V_{\alpha, \beta}\left(\frac{\pi m n g \ell}{c|m h \pm n k|}\right),
\end{aligned}
$$

and

$$
\begin{aligned}
& \mathcal{U}_{\alpha, \beta}^{r}(h, k)=\frac{Q}{2}\left(\frac{Q}{\pi}\right)^{\frac{\alpha+\beta}{2}} \sum_{\substack{(c, h k)=1 \\
c \leq C}} \frac{\mu(c)}{c} \sum_{\substack{m, n \\
(c, m n)=1 \\
m h \neq n k}} \sum_{\substack{a \mid g \\
(e, g)=1}} \mu(a) \frac{\mu(e)}{e} \frac{1}{m^{\frac{1}{2}+\alpha}} \frac{1}{n^{\frac{1}{2}+\beta}} \\
& \sum_{\ell} \frac{1}{\phi(a e \ell)} \sum_{\substack{\bmod a e \ell \\
\psi \neq \psi_{0}}} \psi(m h / g) \bar{\psi}(\mp n k / g) W_{\alpha, \beta}\left(\frac{c|m h \pm n k|}{Q g \ell}\right) V_{\alpha, \beta}\left(\frac{\pi m n g \ell}{c|m h \pm n k|}\right) .
\end{aligned}
$$

The contribution of the non-principal characters will be estimated in the next section, and then we continue the analysis of the main terms arising from $\mathcal{U}_{\alpha, \beta}^{0}(h, k)$ in Section 7 .

\section{THE ERROR TERMS $\mathcal{U}_{\alpha, \beta}^{r}(h, k)$}

Our goal in this section is to establish the estimate

$$
\mathcal{U}_{\alpha, \beta}^{E}=\sum_{h, k \leq Q^{\vartheta}} \frac{\lambda_{h} \overline{\lambda_{k}}}{\sqrt{h k}} \mathcal{U}_{\alpha, \beta}^{r}(h, k) \ll Q^{1+\vartheta+\epsilon} C .
$$

We begin by estimating the contribution of terms arising from (37) with $a \ell \geq Q^{\vartheta+\epsilon} C$ or ael $\geq Q^{12}$. Consider first the contribution of terms where $m h / g \equiv \pm n k / g$ mod ael. Since $m h \neq n k$, these terms satisfy $\max (m h / g, n k / g) \gg$ ael and, since $W_{\alpha, \beta}$ is supported on [1,2], we also have that $c|m h \pm n k| \asymp Q g \ell$, so that (since $a$ divides $g) \max (m, n) \gg Q a \ell /\left(C Q^{\vartheta}\right)$. It follows that for some constant $\tau>\tau_{1}>0$

$$
\left|W_{\alpha, \beta}\left(\frac{c|m h \pm n k|}{Q g \ell}\right) V_{\alpha, \beta}\left(\frac{\pi m n g \ell}{c|m h \pm n k|}\right)\right| \ll \exp \left(-\tau \frac{m n}{Q}\right) \ll \frac{Q}{m n} \exp \left(-\max \left(\frac{\tau_{1} a e \ell}{Q^{2}}, \frac{\tau_{1} a \ell}{C Q^{\vartheta}}\right)\right) \text {. }
$$

This is exponentially small when either $a \ell \geq Q^{\vartheta+\epsilon} C$ or ael $\geq Q^{12}$, and therefore the contribution of these terms is

$$
Q^{1+\epsilon} \sum_{h, k \leq Q^{\vartheta}} \frac{1}{\sqrt{h k}} \sum_{c \leq C} \frac{1}{c} \sum_{\substack{a, e, \ell \\ a e \ell \geq Q^{12}}} \frac{1}{e} \sum_{\substack{m, n \\ m h \neq n k \\ a|g \\ a e \ell|(m h / g \pm n k / g)}} \frac{Q}{(m n)^{\frac{5}{4}}} Q^{-10} \ll Q^{-1} .
$$


Now consider the contribution of terms with $m h / g \not \equiv \pm n k / g \bmod$ ael. These terms contribute

$$
\ll Q^{1+\epsilon} \sum_{h, k \leq Q^{\vartheta}} \frac{1}{\sqrt{h k}} \sum_{c \leq C} \frac{1}{c} \sum_{\begin{array}{c}
a, e, \ell \\
a e \ell \geq Q^{12} \\
\text { or } a \ell \geq C Q^{\vartheta+\epsilon}
\end{array}} \frac{1}{e \phi(a e \ell)} \sum_{m, n} \frac{1}{(m n)^{\frac{1}{2}-\epsilon}} \exp \left(-\frac{\tau m n}{2 Q}-\frac{\tau a \ell}{2 C Q^{\vartheta}}\right) \ll Q^{-1} .
$$
that

Having discarded the contribution from terms with ael $\geq Q^{12}$ or $a \ell \geq C Q^{\vartheta+\epsilon}$, we conclude

$$
\mathcal{U}_{\alpha, \beta}^{E} \ll Q^{1+\epsilon} \sum_{c \leq C} \frac{1}{c} \sum_{\substack{a, e, \ell \\ a e \ell \leq Q^{12} \\ a \ell \leq C Q^{\vartheta+\epsilon}}} \frac{1}{a e^{2} \ell} \sum_{\substack{\psi \rightarrow \infty \\ \psi \neq \psi_{0}}}\left(\left|\mathcal{U}^{+}(c, a, e, \ell ; \psi)\right|+\left|\mathcal{U}_{\alpha, \beta}^{-}(c, a, e, \ell ; \psi)\right|\right)
$$

where

$$
\begin{aligned}
& \mathcal{U}_{\alpha, \beta}^{-}(c, a, e, \ell ; \psi) \\
& =\sum_{\substack{h, k \\
(h k, c)=1}} \frac{\lambda_{h} \overline{\lambda_{k}}}{\sqrt{h k}} \sum_{\substack{m, n \\
(m n, c)=1 \\
a \mid g \\
g, e)=1}} \frac{\psi(m h / g) \bar{\psi}(n k / g)}{m^{\frac{1}{2}+\alpha} n^{\frac{1}{2}+\beta}} W_{\alpha, \beta}\left(\frac{c|m h-n k|}{Q g \ell}\right) V_{\alpha, \beta}\left(\frac{\pi m n g \ell}{c|m h-n k|}\right),
\end{aligned}
$$

and $\mathcal{U}_{\alpha, \beta}^{+}$is defined similarly. To estimate the sums in (38), we divide the sums over $a, e$, and $\ell$ into dyadic blocks $A \leq a<2 A, E \leq e<2 E$, and $L \leq \ell<2 L$. We may assume that $A L \ll C Q^{\vartheta+\epsilon}$ and that $A E L \ll Q^{12}$. After restricting these variables to dyadic blocks, we now focus on the contribution of the sum in (39). The $\mathcal{U}_{\alpha, \beta}^{+}$term can be handled similarly, and in fact is simpler to treat (because while $m h-n k$ can become unusually small in size, $m h+n k$ cannot). The variable $g$ above arises as the gcd of $m h$ and $n k$, and our first task is to separate this variable from $m, h, n$, and $k$ so as to make the sums over those variables independent of each other.

Write $g_{1}=(h, k), g_{2}=(m, n), g_{3}=\left(m / g_{2}, k / g_{1}\right)$, and $g_{4}=\left(n / g_{2}, h / g_{1}\right)$. Then $g=$ $(m h, n k)=g_{1} g_{2} g_{3} g_{4}$. Further, write $h=g_{1} g_{4} H, k=g_{1} g_{3} K, m=g_{2} g_{3} M$ and $n=g_{2} g_{4} N$. There are now a number of coprimality conditions $\left(g_{3}, g_{4}\right)=1,\left(H, g_{3}\right)=1,\left(K, g_{4}\right)=1$, $(H, K)=1,\left(M, g_{4}\right)=1,\left(N, g_{3}\right)=1$, and $(M, N)=1$, and further we must have $a \mid\left(g_{1} g_{2} g_{3} g_{4}\right)$, $\left(g_{1} g_{2} g_{3} g_{4}, e c\right)=1$, and $(M N H K, c)=1$. Thus we may rewrite $\mathcal{U}^{-}(c, a, e, \ell ; \psi)$ as

$$
\sum_{\substack{g_{1}, g_{2}, g_{3}, g_{4} \\ M, N, H, K}}^{*} \frac{\lambda_{g_{1} g_{4} H} \overline{\lambda_{g_{1} g_{3} K}}}{g_{1} \sqrt{g_{3} g_{4} H K}} \frac{\psi(M H) \bar{\psi}(N K)}{\left(g_{2} g_{3} M\right)^{\frac{1}{2}+\alpha}\left(g_{2} g_{4} N\right)^{\frac{1}{2}+\beta}} W_{\alpha, \beta}\left(\frac{c|M H-N K|}{Q \ell}\right) V_{\alpha, \beta}\left(\frac{\pi g_{2}^{2} g_{3} g_{4} M N \ell}{c|M H-N K|}\right) .
$$

Here the $*$ indicates the various coprimality and divisibility conditions mentioned above. 
We now introduce a smooth function $\Psi(x)$ (defined on $[0, \infty)$ ) with $\Psi(x)=1$ for $x \leq 1$ and $\Psi(x)=0$ for $x \geq 2$. Next define, for positive real numbers $x, y$, and $u$

$$
\mathcal{V}_{\alpha, \beta}(x, y ; u)=W_{\alpha, \beta}(|x-y|) \Psi\left(\frac{x L}{C^{2} Q^{\vartheta}}\right) \Psi\left(\frac{y L}{C^{2} Q^{\vartheta}}\right) V_{\alpha, \beta}\left(\frac{u}{|x-y|}\right) .
$$

The support of $W$ guarantees that $1 \leq|x-y| \leq 2$, and therefore $\mathcal{V}_{\alpha, \beta}(x, y ; u)$ decays exponentially in $u$. In (40) we replace the weights

$$
W_{\alpha, \beta}\left(\frac{c|M H-N K|}{Q \ell}\right) V_{\alpha, \beta}\left(\frac{\pi g_{2}^{2} g_{3} g_{4} M N \ell}{c|M H-N K|}\right) \text { with } \mathcal{V}_{\alpha, \beta}\left(\frac{c M H}{Q \ell}, \frac{c N K}{Q \ell} ; \frac{\pi g_{2}^{2} g_{3} g_{4} M N}{Q}\right) \text {. }
$$

The two expressions above are different only if either $M$ or $N$ is at least $C Q$, and in this case the difference is exponentially small because of the rapid decay of $V_{\alpha, \beta}$; therefore we incur a negligible error term in $\mathcal{U}_{\alpha, \beta}^{E}$ in making this replacement. To proceed further, we introduce a three variable Mellin transform of $\mathcal{V}_{\alpha, \beta}$, and discuss its analytic properties.

Lemma 5. Let $s_{1}, s_{2}$ and $z$ denote complex numbers with positive real part. Define

$$
\widetilde{\mathcal{V}}_{\alpha, \beta}\left(s_{1}, s_{2} ; z\right)=\int_{0}^{\infty} \int_{0}^{\infty} \int_{0}^{\infty} \mathcal{V}_{\alpha, \beta}(x, y ; u) x^{s_{1}} y^{s_{2}} u^{z} \frac{d x}{x} \frac{d y}{y} \frac{d u}{u}
$$

Then for any integers $k_{1}, k_{2} \geq 1$ we have

$$
\widetilde{\mathcal{V}}_{\alpha, \beta}\left(s_{1}, s_{2} ; z\right) \ll \frac{\left(1+C^{2} Q^{\vartheta} / L\right)^{k_{1}-1+\operatorname{Re}\left(s_{1}+s_{2}\right)}}{\max \left(\left|s_{1}\right|,\left|s_{2}\right|\right)^{k_{1}}} \frac{1}{|z|^{k_{2}}}
$$

and moreover the following Mellin inversion formula holds: for positive numbers $c_{1}, c_{2}, d$

$$
\mathcal{V}_{\alpha, \beta}(x, y ; u)=\frac{1}{(2 \pi i)^{3}} \int_{\begin{array}{l}
R e\left(s_{1}\right)=c_{1} \\
\operatorname{Re}\left(s_{2}\right)=c_{2} \\
\operatorname{Re}(z)=d
\end{array}} \widetilde{\mathcal{V}}_{\alpha, \beta}\left(s_{1}, s_{2} ; z\right) x^{-s_{1}} y^{-s_{2}} u^{-z} d s_{1} d s_{2} d z .
$$

Proof. Despite the appearance of $|x-y|$, since $W_{\alpha, \beta}$ is supported away from 0 , the function $\mathcal{V}_{\alpha, \beta}(x, y ; u)$ is smooth in all three variables. The estimate on the Mellin transform follows by integrating by parts $k_{2}$ times in the $u$ variable, and $k_{1}$ times in either $x$ or $y$ corresponding to whether $s_{1}$ or $s_{2}$ has larger magnitude, and keeping in mind that $\mathcal{V}_{\alpha, \beta}(x, y ; u)=0$ unless $|x-y| \asymp 1$.

We use the Mellin inversion formula above in (40). Initially we start with $\operatorname{Re}\left(s_{1}\right)=$ $\operatorname{Re}\left(s_{2}\right)=1 / 2+\epsilon$ and $\operatorname{Re}(z)=\epsilon$, where the sums over $M$ and $N$ are absolutely convergent. After relating those sums to $L$-functions attached to non-principal characters, we may move the lines of integration to $\operatorname{Re}\left(s_{1}\right)=\operatorname{Re}\left(s_{2}\right)=\epsilon$. Thus we can bound the quantity in (40) by

$$
\begin{aligned}
Q^{\epsilon} \sum_{g_{1}, g_{2}, g_{3}, g_{4}}^{*} \frac{1}{g_{1} g_{2} g_{3} g_{4}} & \int_{\begin{array}{c}
\operatorname{Re}\left(s_{1}\right)=\epsilon \\
\operatorname{Re}\left(s_{2}\right)=\epsilon \\
\operatorname{Re}(z)=\epsilon
\end{array}}\left|\mathcal{V}_{\alpha, \beta}\left(s_{1}, s_{2} ; z\right)\right|\left|L\left(\frac{1}{2}+\alpha+s_{1}+z, \psi\right) L\left(\frac{1}{2}+\beta+s_{2}+z, \bar{\psi}\right)\right| \\
& \times\left|\sum_{H, K}^{*} \frac{\lambda_{g_{1} g_{4} H} \bar{\lambda}_{g_{1} g_{3} K}}{H^{\frac{1}{2}+s_{1}} K^{\frac{1}{2}+s_{2}}} \psi(H) \bar{\psi}(K)\right|\left|d s_{1} d s_{2} d z\right| .
\end{aligned}
$$


Here the factor $Q^{\epsilon}$ arises from estimating trivially the ratio of the actual sum over $M$ and $N$ (which has coprimality restrictions) and its dominant part (which is the product of $L$ functions given above). Further, in (41), the $*$ over the first sum over $g_{1}, g_{2}, g_{3}, g_{4}$ keeps track of the coprimality conditions of these variables together with the requirement that $a \mid g_{1} g_{2} g_{3} g_{4}$, and the $*$ over the sum over $H$ and $K$ indicates $(H, K)=1$ as well as $\left(H, g_{3} c\right)=\left(K, g_{4} c\right)=1$. Now we use Möbius inversion $\sum_{k \mid(H, K)} \mu(k)=1$ if and only if $(H, K)=1$ in order to separate the variables $H$ and $K$ in the sum in (41). Writing

$$
A(s, \psi ; u, v)=\sum_{(n, v)=1} \frac{\lambda_{u n} \psi(n)}{n^{s}}, \quad \text { and } \quad \bar{A}(s, \psi ; u, v)=\sum_{(n, v)=1} \frac{\bar{\lambda}_{u n} \psi(n)}{n^{s}}
$$

we can bound the sum over $H$ and $K$ in (41) by

$$
\ll \sum_{k} \frac{1}{k}\left|A\left(\frac{1}{2}+s_{1}, \psi ; g_{1} g_{4} k, g_{3} c\right) \bar{A}\left(\frac{1}{2}+s_{2}, \bar{\psi} ; g_{1} g_{3} k, g_{4} c\right)\right| \text {. }
$$

Since $\lambda_{n}=0$ for $n>Q^{\vartheta}$, the above sum over $k$ is just a finite sum.

Inputting these estimates into (39) we find that the contribution of $a, e, \ell$ in dyadic blocks of size $A, E, L$ to $(\underline{38})$ is

$$
\begin{aligned}
& \ll \frac{Q^{1+\epsilon}}{A^{2} E^{2} L} \sum_{A \leq a<2 A} \max _{\substack{u_{1}, u_{2} \\
v_{1}, v_{2}}} \sum_{\substack{E \leq e<2 E \\
L \leq \ell<2 L}} \sum_{\substack{\bmod a e \ell \\
\psi \neq \psi_{0}}} \int_{\substack{\operatorname{Re}\left(s_{1}\right)=\epsilon \\
\operatorname{Re}\left(s_{2}\right)=\epsilon \\
\operatorname{Re}(z)=\epsilon}}\left|\mathcal{V}_{\alpha, \beta}\left(s_{1}, s_{2} ; z\right)\right|\left|L\left(\frac{1}{2}+s_{1}+\alpha+z, \psi\right)\right| \\
& \quad \times\left|L\left(\frac{1}{2}+s_{2}+\beta+z, \bar{\psi}\right)\right|\left|A\left(\frac{1}{2}+s_{1}, \psi ; u_{1}, v_{1}\right) A\left(\frac{1}{2}+s_{2}, \psi ; u_{2}, v_{2}\right)\right|\left|d s_{1} d s_{2} d z\right| .
\end{aligned}
$$

We arrive at this expression by using the max over $u_{1}, u_{2}, v_{1}, v_{2}$ in order to free up the dependencies on $g_{1}, g_{2}, g_{3}, g_{4}$ and $c$. The extra factor of $A$ above arises from the requirement that $g_{1} g_{2} g_{3} g_{4}$ must be a multiple of $a$.

Now we split the integrals over $s_{1}, s_{2}$ into dyadic blocks. From Lemma 5 we see that $\widetilde{\mathcal{V}}_{\alpha, \beta}\left(s_{1}, s_{2} ; z\right)$ decays rapidly in $|z|$. We suppose that $\left|s_{1}\right|$ is of size $S_{1}$ and that this is larger than $\left|s_{2}\right|$. Inputting the bounds of Lemma 5 , the contribution from this dyadic block to the integrals in (43) is (for any natural numbers $k_{1}$ and $k_{2}$ )

$$
\begin{aligned}
\ll Q^{\epsilon} \frac{\left(1+Q^{\vartheta} / L\right)^{k_{1}-1}}{S_{1}^{k_{1}}} \int_{\operatorname{Re}(z)=\epsilon} \frac{1}{|z|^{k_{2}}} \int_{\substack{\operatorname{Re}\left(s_{1}\right)=\operatorname{Re}\left(s_{2}\right)=\epsilon \\
\left|s_{1}\right|,\left|s_{2}\right| \leq 2 S_{1}}}\left|L\left(\frac{1}{2}+s_{1}+\alpha+z, \psi\right) L\left(\frac{1}{2}+s_{2}+\beta+z, \bar{\psi}\right)\right| \\
\times\left|A\left(\frac{1}{2}+s_{1}, \psi ; u_{1}, v_{1}\right) A\left(\frac{1}{2}+s_{2}, \psi ; u_{2}, v_{2}\right)\right|\left|d s_{1} d s_{2} d z\right| .
\end{aligned}
$$

We insert this in (43), and group terms $r=a e$ so that $r$ lies between $A E$ and $4 A E$. We are left with estimating

$$
\begin{aligned}
& \frac{Q^{1+\epsilon}\left(1+Q^{\vartheta} / L\right)^{k_{1}-1}}{A^{2} E^{2} L S_{1}^{k_{1}}} \sum_{A E \leq r<4 A E} \max _{\substack{u_{1}, u_{2} \\
v_{1}, v_{2}}} \sum_{\substack{L \leq \ell<2 L \\
\psi \bmod r \ell \\
\psi \neq \psi_{0}}} \int_{\operatorname{Re}(z)=\epsilon} \frac{1}{|z|^{k_{2}}} \int_{\substack{\operatorname{Re}\left(s_{1}\right)=\operatorname{Re}\left(s_{2}\right)=\epsilon \\
\left|s_{1}\right|,\left|s_{2}\right| \leq 2 S_{1}}}\left|d s_{1} d s_{2} d z\right| \\
& \left|L\left(\frac{1}{2}+s_{1}+\alpha+z, \psi\right) L\left(\frac{1}{2}+s_{2}+\beta+z, \bar{\psi}\right)\right|\left|A\left(\frac{1}{2}+s_{1}, \psi ; u_{1}, v_{1}\right) A\left(\frac{1}{2}+s_{2}, \psi ; u_{2}, v_{2}\right)\right| .
\end{aligned}
$$


At this stage we appeal to the following consequence of the hybrid large sieve inequality.

Proposition 1. Given a character $\chi$, put

$$
M(s, \chi)=\sum_{n \leq N} a(n) \chi(n) n^{-s}
$$

where $a(n) \ll n^{\epsilon}$. Let $\sigma \geq 1 / 2$ be a real number, and $z$ a complex number with $\operatorname{Re}(z)>0$. Let $k$ be a positive integer, and $Q \geq 2$ and $T \geq 2$ be real numbers. Then

$$
\begin{aligned}
\sum_{q \leq Q} \sum_{\substack{\bmod q k \\
\chi \neq \chi_{0}}}\left(\int_{-T}^{T} \mid\right. & L(\sigma+i t+z, \chi) M(\sigma+i t, \chi) \mid d t)^{2} \\
& \ll(k Q T N(1+|z|))^{\epsilon}\left(k Q^{2} T^{2}(1+|z|)^{\frac{1}{2}}+Q N T(1+|z|)^{\frac{1}{2}} k^{\frac{1}{2}}\right) .
\end{aligned}
$$

Postponing the proof of the proposition for the moment, we first finish the treatment of $\mathcal{U}_{\alpha, \beta}^{E}$. From Proposition 1 we may bound the quantity in (44) by

$$
\begin{aligned}
\ll \frac{Q^{1+\epsilon}\left(1+Q^{\vartheta} / L\right)^{k_{1}-1}}{A^{2} E^{2} L S_{1}^{k_{1}}} \sum_{A E \leq r<4 A E} \int_{\operatorname{Re}(z)=\epsilon} \frac{1}{|z|^{k_{2}}} & \left(A E L Q S_{1}(1+|z|)\right)^{\epsilon}(1+|z|)^{\frac{1}{2}} \\
& \times\left(A E L^{2} S_{1}^{2}+A^{\frac{1}{2}} E^{\frac{1}{2}} L Q^{\vartheta} S_{1}\right)|d z| .
\end{aligned}
$$

Taking $k_{2}=2$, this simplifies to

$$
\ll \frac{Q^{1+\epsilon}\left(1+Q^{\vartheta} / L\right)^{k_{1}-1}}{A^{2} E^{2} L S_{1}^{k_{1}}}\left(A E L Q S_{1}\right)^{\epsilon}\left(A^{2} E^{2} L^{2} S_{1}^{2}+A^{\frac{3}{2}} E^{\frac{3}{2}} L Q^{\vartheta} S_{1}\right) .
$$

Take above $k_{1}=1$ if $S_{1} \leq 1+Q^{\vartheta} / L$ and take $k_{1}=3$ if $S_{1}$ is larger. Then, after summing over all the possible dyadic sizes of $S_{1}$, the above is seen to be $\ll(A E L Q)^{\epsilon} Q\left(L+Q^{\vartheta}\right)$. Since we may restrict attention to $L \leq A L \ll C Q^{\vartheta+\epsilon}$ and $A E L \ll Q^{12}$, it follows that $\mathcal{U}_{\alpha, \beta}^{E} \ll C Q^{1+\vartheta+\epsilon}$ as needed.

Proof of Proposition 1. We may clearly assume that the coefficients $a(n)$ are non-zero only when $(n, k)=1$. We first pass from all non-principal characters $\bmod q k$ to primitive characters. Suppose $\chi \bmod q k$ is induced by a primitive character $\tilde{\chi} \bmod \widetilde{q} \widetilde{k}$ where $(k, \widetilde{q})=1$ and $\widetilde{k}$ is composed only of primes dividing $k$. We write $q=d \widetilde{q} \widetilde{k} /(k, \widetilde{k})$ for some integer $d$. Then we can recast our sum as

$$
\ll(Q k)^{\epsilon} \sum_{p|\widetilde{k} \Longrightarrow p| k} \sum_{\substack{\widetilde{k} \\ \Longrightarrow(k, \widetilde{k}) / \widetilde{k} \widetilde{q} \leq Q(k, \widetilde{k}) /(\widetilde{k} d)}} \sum_{\substack{(k, \widetilde{q})=1 \\ \widetilde{k} \widetilde{q}>1}}^{*}\left(\int_{-T}^{T}\left|L(\sigma+i t+z, \widetilde{\chi}) M_{d}(\sigma+i t, \widetilde{\chi})\right| d t\right)^{2} .
$$

Here the factor $(Q k)^{\epsilon}$ accounts for the difference between $L(s, \chi)$ and $L(s, \tilde{\chi})$, and $M_{d}$ indicates that the Dirichlet polynomial $M$ is restricted to terms coprime to $d$. 
Given $\widetilde{k}$ and $d$, we now bound the sum over $\widetilde{q}$ and $\widetilde{\chi}$ using the hybrid large sieve (see Theorem 9.12 of [11]). We do this in two different ways, depending on whether $T>Q(k, \widetilde{k}) / d$ or not. In the first case, by Cauchy-Schwarz

$$
\left(\int_{-T}^{T}\left|L(\sigma+i t+z, \widetilde{\chi}) M_{d}(\sigma+i t, \tilde{\chi})\right| d t\right)^{2} \leq\left(\int_{-T}^{T}|L(\sigma+i t+z, \widetilde{\chi})|^{2} d t\right)\left(\int_{-T}^{T}\left|M_{d}(\sigma+i t, \widetilde{\chi})\right|^{2} d t\right) .
$$

Since $\tilde{\chi}$ is not principal, approximating the $L$-function by a Dirichlet polynomial of length $\ll \sqrt{(1+|z|) T \widetilde{q} \widetilde{k}} \ll \sqrt{1+|z|} T$ we may bound the first integral on the right side by $\ll$ $\left.(Q k)^{\epsilon}(1+|z|)\right)^{\frac{1}{2}+\epsilon} T^{1+\epsilon}$. Therefore we obtain the bound

$$
\ll(Q k)^{\epsilon}(1+|z|)^{\frac{1}{2}+\epsilon} T^{1+\epsilon} \sum_{\substack{\widetilde{q} \leq Q(k, \widetilde{k}) /(\widetilde{k} d) \\(k, \tilde{q})=1}} \sum_{\widetilde{\chi} \bmod \widetilde{q} \widetilde{k}}^{*} \int_{-T}^{T}\left|M_{d}(\sigma+i t, \widetilde{\chi})\right|^{2} d t,
$$

which by the hybrid large sieve is

$$
\ll(Q k N)^{\epsilon}(1+|z|)^{\frac{1}{2}+\epsilon} T^{1+\epsilon}\left(Q^{2} T \frac{(k, \widetilde{k})^{2}}{\widetilde{k} d^{2}}+N\right) .
$$

Summing this over all the possible $\widetilde{k}$ and $1 \leq d \leq Q(k, \widetilde{k}) / \widetilde{k}$ we arrive at the estimate

$$
\begin{aligned}
& \ll(Q k N T(1+|z|))^{\epsilon} \sum_{\substack{p \\
p \mid \widetilde{k}} p \mid k}\left(Q^{2} T^{2}(1+|z|)^{\frac{1}{2}} \frac{(k, \widetilde{k})^{2}}{\widetilde{k}}+Q N T(1+|z|)^{\frac{1}{2}} \frac{(k, \widetilde{k})}{\widetilde{k}}\right) \\
& \ll(Q k N T(1+|z|))^{\epsilon}\left(k Q^{2} T^{2}(1+|z|)^{\frac{1}{2}}+Q N T(1+|z|)^{\frac{1}{2}}\right) .
\end{aligned}
$$

Now we consider the case where $T \leq Q(k, \widetilde{k}) / d$, where by Cauchy-Schwarz we must estimate

$$
T \sum_{\substack{\widetilde{q} \leq Q(k, \widetilde{k}) /(\widetilde{k} d) \\(k, \tilde{q})=1}} \sum_{\tilde{\chi} \bmod \widetilde{q} \widetilde{k}}^{*} \int_{-T}^{T}\left|L\left(\frac{1}{2}+i t+z, \widetilde{\chi}\right) M_{d}(\sigma+i t, \widetilde{\chi})\right|^{2} d t .
$$

An application of the hybrid large sieve bounds the above by

$$
\ll(Q k N T(1+|z|))^{\epsilon} T\left(Q^{2} T \frac{(k, \widetilde{k})^{2}}{\widetilde{k} d^{2}}+N\left(\frac{Q T(k, \widetilde{k})(1+|z|)}{d}\right)^{\frac{1}{2}}\right),
$$

and summing this over $\widetilde{k}$ and $d \leq Q(k, \widetilde{k}) / \max (T, \widetilde{k})$ we obtain the estimate

$$
\begin{aligned}
& \ll(Q k N T(1+|z|))^{\epsilon} \sum_{\substack{\tilde{k}} p \mid k}\left(Q^{2} T^{2} \frac{(k, \widetilde{k})^{2}}{\widetilde{k}}+Q N T\left(\frac{T(1+|z|)(k, \widetilde{k})}{\max (T, \widetilde{k})}\right)^{\frac{1}{2}}\right) \\
& \ll(Q k N T(1+|z|))^{\epsilon}\left(Q^{2} T^{2} k+Q N T k^{\frac{1}{2}}(1+|z|)^{\frac{1}{2}}\right) .
\end{aligned}
$$


Since either (46) or (47) applies, the proposition follows.

\section{ThE PRINCIPAL CHARACTER CONTRIBUtion}

In this section we work on the principal character contribution arising from (36). For technical reasons that will become clear later, we introduce a small smoothing of the weight functions appearing in (36). Let $\delta$ denote a small parameter, which we shall choose as $\delta=Q^{-10}$, and define for positive real numbers $x, y$, and $t$

$$
\mathcal{W}_{\alpha, \beta}(x, y ; t)=\frac{1}{2 \delta} \int_{-\delta}^{\delta} W_{\alpha, \beta}\left(\left|x \pm e^{\xi} y\right|\right) V_{\alpha, \beta}\left(\frac{t}{\left|x \pm e^{\xi} y\right|}\right) d \xi
$$

A small calculation, using the rapid decay of $V_{\alpha, \beta}$ and that $W_{\alpha, \beta}$ is supported on [1,2], shows that (for some constant $\tau>0$ )

$$
\mathcal{W}_{\alpha, \beta}(x, y ; t)=W_{\alpha, \beta}(|x \pm y|) V_{\alpha, \beta}\left(\frac{t}{|x \pm y|}\right)+O\left(\delta y e^{-\tau t}\right)
$$

Therefore we may recast (36) as

$$
\begin{aligned}
\mathcal{U}_{\alpha, \beta}^{0}(h, k)=\frac{Q}{2}\left(\frac{Q}{\pi}\right)^{\frac{\alpha+\beta}{2}} & \sum_{\substack{(c, h k)=1 \\
c \leq C}} \frac{\mu(c)}{c} \sum_{\substack{m, n \\
(c, m n)=1 \\
m h \neq n k}} \sum_{\substack{a \mid g \\
(e, g)=1}} \mu(a) \frac{\mu(e)}{e} \frac{1}{m^{\frac{1}{2}+\alpha}} \frac{1}{n^{\frac{1}{2}+\beta}} \\
& \sum_{\substack{\ell \\
\left(a e \ell, m n h k / g^{2}\right)=1}} \frac{1}{\phi(a e \ell)} \mathcal{W}_{\alpha, \beta}\left(\frac{c m h}{Q g \ell}, \frac{c n k}{Q g \ell} ; \frac{\pi m n}{Q}\right)+O\left(Q^{-1}\right) .
\end{aligned}
$$

Treating $c, m, n, a$ and $e$ as given, we work on simplifying the sum over $\ell$ in (50). By Mellin inversion, the sum over $\ell$ may be written as

$$
\sum_{\substack{\left.\ell \\, m n h k / g^{2}\right)=1}} \frac{1}{\phi(a e \ell)} \frac{1}{2 \pi i} \int_{(\epsilon)} \ell^{-w} \widetilde{\mathcal{W}}_{\alpha, \beta}(w) d w
$$

where (suppressing the dependence of $\widetilde{\mathcal{W}}_{\alpha, \beta}$ on $c, m, n, h, k, g$ )

$$
\widetilde{\mathcal{W}}_{\alpha, \beta}(w)=\int_{0}^{\infty} \mathcal{W}_{\alpha, \beta}\left(\frac{c m h}{Q g x}, \frac{c n k}{Q g x} ; \frac{\pi m n}{Q}\right) x^{w} \frac{d x}{x} .
$$

Lemma 6. Let $s$ be a complex number with $\operatorname{Re}(s)>0$, and let $u$ and $v$ be coprime natural numbers. Then

where

$$
\sum_{\substack{\ell=1 \\(\ell, v)=1}}^{\infty} \frac{1}{\phi(u \ell) \ell^{s}}=\frac{1}{\phi(u)} \zeta(1+s) R(s ; u, v)
$$

$$
R(s ; u, v)=\prod_{p \mid v}\left(1-\frac{1}{p^{s+1}}\right) \prod_{p \nmid u v}\left(1+\frac{1}{p^{s+1}(p-1)}\right)
$$


converges absolutely in $\operatorname{Re}(s)>-1$.

Proof. This is a simple verification using Euler products.

We use Lemma 6 in (51) and move the line of integration to the $(-\epsilon)$ line. Computing the residue of the pole at $w=0$, the quantity in (51) becomes

$$
\frac{\widetilde{\mathcal{W}}_{\alpha, \beta}(0)}{\phi(a e)} R\left(0 ; a e, m n h k / g^{2}\right)+\frac{1}{2 \pi i \phi(a e)} \int_{(-\epsilon)} \zeta(1+w) R\left(w ; a e, m n h k / g^{2}\right) \widetilde{\mathcal{W}}_{\alpha, \beta}(w) d w .
$$

Using this in (50), the quantity $\mathcal{U}_{\alpha, \beta}^{0}(h, k)$ splits into $\mathcal{U}_{\alpha, \beta}^{1}(h, k)+\mathcal{U}_{\alpha, \beta}^{2}(h, k)+O\left(Q^{-1}\right)$, with the first term denoting the contribution of the residue at $w=0$, and the second term denoting the remaining integral.

7.1. The contribution of $\mathcal{U}_{\alpha, \beta}^{1}(h, k)$. Here we show that $\mathcal{U}_{\alpha, \beta}^{1}(h, k)$ cancels out the contribution of $\mathcal{L}_{\alpha, \beta}^{0}(h, k)$. First we perform the sum over $a$ and $e$. Note that $\widetilde{\mathcal{W}}_{\alpha, \beta}$ is independent of $a$ and $e$, and for any $w$ with $\operatorname{Re}(w)>-1$ we have (with a small calculation using that $a \mid g$ and $(e, g)=1$ so that $(a, e)=1)$

$$
\begin{aligned}
\sum_{\substack{\left.a \mid g \\
m n h k / g^{2}\right)=1}} \mu(a) \sum_{(e, m n h k / g)=1} \frac{\mu(e)}{e \phi(a e)} R\left(w ; a e, m n h k / g^{2}\right) & =\prod_{p \mid m n h k / g^{2}}\left(1-\frac{1}{p^{1+w}}\right) \\
& \times \prod_{\substack{p \mid g \\
p \nmid m n h k / g^{2}}}\left(1+\frac{1}{p^{1+w}(p-1)}-\frac{1}{p-1}\right) \prod_{p \nmid m n h k / g}\left(1+\frac{p^{-w}-1}{p(p-1)}\right) .
\end{aligned}
$$

Evaluating this at $w=0$, we see that the sum of the first term in (53) over the relevant $a$ and $e$ is

$$
\widetilde{\mathcal{W}}_{\alpha, \beta}(0) \prod_{p \mid m n h k / g^{2}}\left(1-\frac{1}{p}\right) \prod_{\substack{p \mid g \\ p \nmid m n h k / g^{2}}}\left(1-\frac{1}{p}\right)=\widetilde{\mathcal{W}}_{\alpha, \beta}(0) \frac{\phi(m n h k)}{m n h k} .
$$

Now from (52) and a change of variables we find

$$
\widetilde{\mathcal{W}}_{\alpha, \beta}(0)=2 \int_{0}^{\infty} W_{\alpha, \beta}\left(\frac{x}{Q}\right) V_{\alpha, \beta}\left(\frac{\pi m n}{x}\right) \frac{d x}{x}
$$

where the factor 2 arises from our convention that $|m h \pm n k|$ indicates a sum over both possible signs. Combining this with our calculations above, we conclude that

$$
\begin{aligned}
& \mathcal{U}_{\alpha, \beta}^{1}(h, k)=Q\left(\frac{Q}{\pi}\right)^{\frac{\alpha+\beta}{2}} \sum_{\substack{(c, h k)=1 \\
c \leq C}} \frac{\mu(c)}{c} \sum_{\substack{m, n \\
(c, m n)=1 \\
m h \neq n k}} \frac{1}{m^{\frac{1}{2}+\alpha} n^{\frac{1}{2}+\beta}} \frac{\phi(m n h k)}{m n h k} \\
&\left(\int_{0}^{\infty} W_{\alpha, \beta}\left(\frac{x}{Q}\right) V_{\alpha, \beta}\left(\frac{\pi m n}{x}\right) \frac{d x}{x}\right) .
\end{aligned}
$$

Note that this cancels (up to an acceptable error) with our expression for $\mathcal{L}_{\alpha, \beta}^{0}(h, k)$ in (29)). 
7.2. The terms $\mathcal{U}_{\alpha, \beta}^{2}(h, k)$. Write

$$
R_{1}(w ; u, v)=\prod_{p \mid v}\left(1-\frac{1}{p^{1+w}}\right) \prod_{\substack{p \mid u \\ p \nmid v}}\left(1+\frac{1}{p^{1+w}(p-1)}-\frac{1}{p-1}\right) \prod_{p \nmid u v}\left(1+\frac{p^{-w}-1}{p(p-1)}\right),
$$

so that the right hand side of (54) equals $R_{1}\left(w ; g, m n h k / g^{2}\right)$. Using the notation (55), together with (54), (53), (51) and finally (50), we see that

$$
\begin{aligned}
\mathcal{U}_{\alpha, \beta}^{2}(h, k)=\frac{Q}{2}\left(\frac{Q}{\pi}\right)^{\frac{\alpha+\beta}{2}} & \sum_{\substack{c \leq C \\
(c, h k)=1}} \frac{\mu(c)}{c} \sum_{\substack{m, n \\
(c, m n)=1 \\
m h \neq n k}} \frac{1}{m^{\frac{1}{2}+\alpha} n^{\frac{1}{2}+\beta}} \\
& \times \frac{1}{2 \pi i} \int_{(-\epsilon)} \zeta(1+w) R_{1}\left(w ; g, m n h k / g^{2}\right) \widetilde{\mathcal{W}}_{\alpha, \beta}(w) d w .
\end{aligned}
$$

Recall that $\widetilde{\mathcal{W}}_{\alpha, \beta}$ was defined in (52), and that it depends on $c, m, n, h, k$, and $g$.

We now express $\widetilde{\mathcal{W}}_{\alpha, \beta}(w)$ in a more useful form, making explicit the dependencies on $c$, $m, n, h, k, g$, before continuing with the evaluation of (56). With the change of variable $y=Q g x /\left(c\left|m h \pm e^{\xi} n k\right|\right)$ (the denominator can be zero for at most one value of $\xi$, and this is irrelevant for the integral over $\xi$ below) we obtain

$$
\widetilde{\mathcal{W}}_{\alpha, \beta}(w)=\left(\frac{1}{2 \delta} \int_{-\delta}^{\delta}\left(\frac{c\left|m h \pm e^{\xi} n k\right|}{Q g}\right)^{w} d \xi\right)\left(\int_{0}^{\infty} W_{\alpha, \beta}(1 / y) V_{\alpha, \beta}\left(\frac{\pi m n}{Q} y\right) y^{w} \frac{d y}{y}\right) .
$$

Using (12), we see that the second factor above is

$$
\begin{aligned}
& =\frac{1}{2 \pi i} \int_{(1)} \widetilde{V}_{\alpha, \beta}(s)\left(\frac{Q}{\pi m n}\right)^{s}\left(\int_{0}^{\infty} W_{\alpha, \beta}(1 / y) y^{w-s} \frac{d y}{y}\right) \frac{d s}{s} \\
& =\frac{1}{2 \pi i} \int_{(1)} \widetilde{V}_{\alpha, \beta}(s)\left(\frac{Q}{\pi m n}\right)^{s} \widetilde{W}_{\alpha, \beta}(s-w) \frac{d s}{s}
\end{aligned}
$$

We point out the difference between $\widetilde{\mathcal{W}}_{\alpha, \beta}$ and $\widetilde{W}_{\alpha, \beta}$ (the Mellin transform of $W_{\alpha, \beta}$ ), which we hope will not cause confusion.

To handle the first factor in (57) we require the following integration formula, which is where the smoothing of the weight functions introduced above will be useful. Given complex numbers $z$ and $w$, we define

$$
\mathcal{H}(w, z)=2^{z} \sin \left(\frac{\pi z}{2}\right) \Gamma(1-z) \frac{\Gamma\left(\frac{w}{2}\right) \Gamma\left(\frac{z-w}{2}\right)}{\Gamma\left(\frac{1-w}{2}\right) \Gamma\left(\frac{1-z+w}{2}\right)}=\sqrt{\pi} \frac{\Gamma\left(\frac{1-z}{2}\right) \Gamma\left(\frac{w}{2}\right) \Gamma\left(\frac{z-w}{2}\right)}{\Gamma\left(\frac{z}{2}\right) \Gamma\left(\frac{1-w}{2}\right) \Gamma\left(\frac{1-z+w}{2}\right)} .
$$

The variables $w$ and $z$ in (59) and the following proposition are temporary, and in particular, we warn that $w$ is not the variable of (57). 
Proposition 2. Let $z=x+i y$ be a complex number with $\operatorname{Re}(z)=x>0$. Then for any $0<c<x$, and $r>0$ with $r \neq 1$, we have

$$
|1 \pm r|^{-z}=|1+r|^{-z}+|1-r|^{-z}=\frac{1}{2 \pi i} \int_{(c)} \mathcal{H}(w, z) r^{-w} d w
$$

Therefore, for any $\delta>0$,

$$
\frac{1}{2 \delta} \int_{-\delta}^{\delta}\left|1 \pm e^{\xi} r\right|^{-z} d \xi=\frac{1}{2 \pi i} \int_{(c)} \mathcal{H}(w, z) r^{-w} \frac{e^{\delta w}-e^{-\delta w}}{2 \delta w} d w
$$

and the integral above converges absolutely for $x<1$.

Proof. We first establish (60), whose proof is similar to the standard proofs of Perron's formula, see [14. If $r>1$ then move the line of integration in (60) to the right. We encounter simple poles at $w=z+2 k$ for all non-negative integers $k$. Noting that the contour is oriented clockwise, the contribution of the residue at $w=z+2 k$ equals

$$
\sqrt{\pi} \frac{\Gamma\left(\frac{1-z}{2}\right) \Gamma\left(\frac{z}{2}+k\right)}{\Gamma\left(\frac{z}{2}\right) \Gamma\left(\frac{1-z}{2}-k\right) \Gamma\left(k+\frac{1}{2}\right)}\left(2 \frac{(-1)^{k}}{k !} r^{-z-2 k}\right)=2 r^{-z-2 k} \frac{z(z+1) \ldots(z+2 k-1)}{(2 k) !} .
$$

Summing this over all non-negative integers $k$ gives

$$
2 r^{-z} \sum_{k=0}^{\infty} r^{-2 k} \frac{z(z+1) \ldots(z+2 k-1)}{(2 k) !}=(r+1)^{-z}+(r-1)^{-z} \text {. }
$$

If $r<1$ then we move the line of integration to the left, encountering poles at $w=-2 k$ for non-negative integers $k$, and argue similarly. The identity (61) follows by integrating (60).

The integral in (60) is only conditionally convergent, and should be interpreted symmetrically as $\lim _{T \rightarrow \infty} \int_{c-i T}^{c+i T}$. The variant given in (61) has the advantage of giving absolutely convergent integrals. To see this, we use Stirling's formula to conclude that $\mathcal{H}(w, z) \ll$ $(1+|w|)^{x-1}$ for $w$ bounded away from the poles of $\mathcal{H}(w, z)$. Therefore if $x<1$, the integral in (61) converges absolutely.

We now return to the first factor in (57), using Proposition 2 to give an expression for it. Thus (recalling $\operatorname{Re}(w)=-\epsilon$ ) we have

$$
\begin{aligned}
\frac{1}{2 \delta} \int_{-\delta}^{\delta}\left(\frac{c\left|m h \pm e^{\xi} n k\right|}{Q g}\right)^{w} d \xi & =\left(\frac{c m h}{Q g}\right)^{w} \frac{1}{2 \delta} \int_{-\delta}^{\delta}\left|1 \pm e^{\xi} \frac{n k}{m h}\right|^{w} d \xi \\
& =\left(\frac{c m h}{Q g}\right)^{w} \frac{1}{2 \pi i} \int_{(\epsilon / 2)} \mathcal{H}(z,-w)\left(\frac{m h}{n k}\right)^{z} \frac{e^{\delta z}-e^{-\delta z}}{2 \delta z} d z
\end{aligned}
$$


Combining this with (58), we conclude that

$$
\begin{aligned}
\widetilde{\mathcal{W}}_{\alpha, \beta}(w)=\frac{1}{(2 \pi i)^{2}} \int_{\substack{\operatorname{Re}(s)=1 \\
\operatorname{Re}(z)=\epsilon / 2}} \widetilde{V}_{\alpha, \beta}(s) \widetilde{W}_{\alpha, \beta}(s-w) \mathcal{H}(z,-w) \\
\quad \times\left(\frac{Q}{\pi m n}\right)^{s}\left(\frac{c m h}{g Q}\right)^{w}\left(\frac{h m}{n k}\right)^{z} \frac{e^{\delta z}-e^{-\delta z}}{2 \delta z} \frac{d s}{s} d z .
\end{aligned}
$$

We use (62) in the formula (56), and bring in the sums over $m$ and $n$. The condition that $m h \neq n k$ can now be discarded with a negligible error of $\ll Q^{1+\epsilon}(h k)^{\epsilon}$ by moving the line of integration in $s$ to $\operatorname{Re}(s)=4 \epsilon$ here. Using this to reintroduce the terms $m h=n k$ in (56), we define (given $z$ with $\operatorname{Re}(z)=\epsilon / 2$, and $w$ with $\operatorname{Re}(w)=-\epsilon$ )

$$
\mathcal{K}(s, z, w ; c, h, k)=\sum_{\substack{m, n \\(m n, c)=1}} \frac{1}{m^{\frac{1}{2}+\alpha}} \frac{1}{n^{\frac{1}{2}+\beta}} \frac{1}{(m n)^{s}} \frac{m^{z+w}}{g^{w} n^{z}} R_{1}\left(w ; g, m n h k / g^{2}\right) .
$$

For $z$ and $w$ as above, the sums over $m$ and $n$ are absolutely convergent for $\operatorname{Re}(s)>\frac{1}{2}+4 \epsilon$ say. By considering Euler products, we may write

$$
\mathcal{K}(s, z, w ; c, h, k)=\zeta\left(\frac{1}{2}+\alpha+s-z-w\right) \zeta\left(\frac{1}{2}+\beta+s+z\right) \mathcal{K}_{1}(s, z, w ; c, h, k),
$$

where $\mathcal{K}_{1}$ is absolutely convergent for $\operatorname{Re}(s)>4 \epsilon$.

Thus

$$
\begin{aligned}
\mathcal{U}_{\alpha, \beta}^{2}(h, k)= & \frac{Q}{2}\left(\frac{Q}{\pi}\right)^{\frac{\alpha+\beta}{2}} \sum_{\substack{c \leq C \\
(c, h k)=1}} \frac{\mu(c)}{c} \frac{1}{(2 \pi i)^{2}} \int_{\substack{\operatorname{Re}(w)=-\epsilon \\
\operatorname{Re}(z)=\epsilon / 2}} \zeta(1+w) \mathcal{H}(z,-w) \frac{c^{w} h^{w+z}}{Q^{w} k^{z}} \\
& \times \frac{1}{2 \pi i} \int_{\operatorname{Re}(s)=1}\left(\frac{Q}{\pi}\right)^{s} \widetilde{V}_{\alpha, \beta}(s) \widetilde{W}_{\alpha, \beta}(s-w) \mathcal{K}(s, z, w ; c, h, k)\left(\frac{e^{\delta z}-e^{-\delta z}}{2 \delta z}\right) \frac{d s}{s} d z d w .
\end{aligned}
$$

Now we use (64), and move the line of integration in $s$ to $\operatorname{Re}(s)=4 \epsilon$. In doing so, we encounter poles at $s=\frac{1}{2}+z+w-\alpha$, and $s=\frac{1}{2}-\beta-z$, and the remaining integrals with $\operatorname{Re}(s)=4 \epsilon$ may be bounded by $\ll Q^{1+\epsilon}(h k)^{\epsilon}$.

Next we examine the contribution of the pole at $s=\frac{1}{2}-\beta-z$ (which will turn out to be unimportant). This contributes to $\mathcal{U}_{\alpha, \beta}^{2}(h, k)$ an amount

$$
\begin{aligned}
& \frac{Q}{2}\left(\frac{Q}{\pi}\right)^{\frac{\alpha+\beta}{2}} \sum_{\substack{c \leq C \\
(c, h k)=1}} \frac{\mu(c)}{c} \frac{1}{(2 \pi i)^{2}} \int_{\substack{\operatorname{Re}(w)=-\epsilon \\
\operatorname{Re}(z)=\epsilon / 2}} \zeta(1+w) \mathcal{H}(z,-w) \frac{c^{w} h^{w+z}}{Q^{w} k^{z}} \zeta(1+\alpha-\beta-2 z-w) \\
& \times \mathcal{K}_{1}\left(\frac{1}{2}-\beta-z, z, w ; c, h, k\right)\left(\frac{Q}{\pi}\right)^{\frac{1}{2}-\beta-z} \frac{\widetilde{V}_{\alpha, \beta}\left(\frac{1}{2}-\beta-z\right)}{\frac{1}{2}-\beta-z} \widetilde{W}_{\alpha, \beta}\left(\frac{1}{2}-\beta-z-w\right)\left(\frac{e^{\delta z}-e^{-\delta z}}{2 \delta z}\right) d z d w .
\end{aligned}
$$


Here we move the line of integration in $z$ to $\operatorname{Re}(z)=1 / 2-\epsilon$ and then bound this contribution by $\ll Q^{1+\epsilon} \sqrt{h / k}$, which is acceptable as

$$
\sum_{h, k} \frac{\left|\lambda_{h} \lambda_{k}\right|}{\sqrt{h k}} Q^{1+\epsilon} \sqrt{h / k} \ll Q^{1+\vartheta+\epsilon} .
$$

Now we turn to the contribution of the important pole at $s=\frac{1}{2}+z+w-\alpha$, which accounts for new main terms to go with the diagonal contribution $\mathcal{D}_{\alpha, \beta}(h, k)$. The contribution of this pole to $\mathcal{U}_{\alpha, \beta}^{2}(h, k)$ is

$$
\begin{aligned}
& \frac{Q}{2}\left(\frac{Q}{\pi}\right)^{\frac{\alpha+\beta}{2}} \sum_{\substack{c \leq C \\
(c, h k)=1}} \frac{\mu(c)}{c} \frac{1}{(2 \pi i)^{2}} \int_{\substack{\operatorname{Re}(w)=-\epsilon \\
\operatorname{Re}(z)=\epsilon / 2}} \zeta(1+w) \mathcal{H}(z,-w) \frac{c^{w} h^{w+z}}{Q^{w} k^{z}} \zeta(1+\beta-\alpha+2 z+w) \\
& \quad \times \mathcal{K}_{1}\left(\frac{1}{2}-\alpha+z+w, z, w ; c, h, k\right)\left(\frac{Q}{\pi}\right)^{\frac{1}{2}+z+w-\alpha} \frac{\widetilde{V}_{\alpha, \beta}\left(\frac{1}{2}-\alpha+z+w\right)}{\frac{1}{2}-\alpha+z+w} \widetilde{W}_{\alpha, \beta}\left(\frac{1}{2}+z-\alpha\right) d z d w .
\end{aligned}
$$

Now we move the line of integration in $z$ to $\operatorname{Re}(z)=-\frac{1}{2}+3 \epsilon$, passing through a pole at $z=(\alpha-\beta-w) / 2$. The remaining integral contributes $\ll Q^{1+\epsilon} \sqrt{k / h}$ (which as before is acceptable), while the residue of this pole gives

$$
\begin{aligned}
\frac{Q}{2}\left(\frac{Q}{\pi}\right)^{\frac{\alpha+\beta}{2}} \sum_{\substack{c \leq C \\
(c, h \bar{h})=1}} \frac{\mu(c)}{c} & \frac{1}{2 \pi i} \int_{\operatorname{Re}(w)=-\epsilon} \zeta(1+w) \mathcal{H}\left(\frac{\alpha-\beta-w}{2},-w\right) \frac{c^{w} h^{\frac{\alpha-\beta+w}{2}}}{Q^{w} k^{\frac{\alpha-\beta-w}{2}}}\left(\frac{Q}{\pi}\right)^{\frac{1-\alpha-\beta+w}{2}} \\
& \times \mathcal{K}_{1}\left(\frac{1-\alpha-\beta+w}{2}, \frac{\alpha-\beta-w}{2}, w ; c, h, k\right) \frac{\widetilde{V}_{\alpha, \beta}((1-\alpha-\beta+w) / 2)}{(1-\alpha-\beta+w)} \\
& \times \widetilde{W}_{\alpha, \beta}\left(\frac{1-\alpha-\beta-w}{2}\right)(1+O(|w| \delta)) d w .
\end{aligned}
$$

Since $\delta=Q^{-10}$ the error term above is negligible, and making the substitution $w=-1+$ $\alpha+\beta-2 s$ and see that the above equals

$$
\begin{aligned}
-\frac{Q^{2-\frac{\alpha+\beta}{2}}}{2 \pi^{\frac{\alpha+\beta}{2}}} & \sum_{\substack{c \leq C \\
(c, h k)=1}} \frac{\mu(c)}{c} \frac{1}{2 \pi i} \int_{\left(-\frac{1}{2}+\epsilon\right)} \zeta(\alpha+\beta-2 s) \mathcal{H}\left(\frac{1}{2}-\beta+s, 1-\alpha-\beta+2 s\right) \\
& \times c^{-1+\alpha+\beta-2 s}\left(\frac{Q \pi}{h k}\right)^{s} \frac{h^{\alpha-\frac{1}{2}}}{k^{\frac{1}{2}-\beta}} \mathcal{K}_{1}\left(-s, \frac{1}{2}-\beta+s,-2 s-1+\alpha+\beta ; c, h, k\right) \\
& \times \widetilde{V}_{\alpha, \beta}(-s) \widetilde{W}_{\alpha, \beta}(1-\alpha-\beta+s) \frac{d s}{s} .
\end{aligned}
$$

Summarizing, the expression in (66) evaluates $\mathcal{U}_{\alpha, \beta}^{2}(h, k)$ up to admissible error terms. 


\section{Proof of the Main Theorem}

Putting together our work from the previous sections, we find that the main terms contributing to $\Delta_{\alpha, \beta}(h, k)$ are

$$
\mathcal{D}_{\alpha, \beta}(h, k)+\mathcal{D}_{-\beta,-\alpha}(h, k)+\mathcal{U}_{\alpha, \beta}^{2}(h, k)+\mathcal{U}_{-\beta,-\alpha}^{2}(h, k) .
$$

Here the quantity $\mathcal{D}_{\alpha, \beta}(h, k)$ is evaluated in (26) , and the main term for $\mathcal{U}_{\alpha, \beta}^{2}(h, k)$ is given by (66), and similar expressions hold for $\mathcal{D}_{-\beta,-\alpha}(h, k)$ and $\mathcal{U}_{-\beta,-\alpha}^{2}(h, k)$. We now show that the terms $\mathcal{D}_{\alpha, \beta}(h, k)$ and $\mathcal{U}_{-\beta,-\alpha}(h, k)$ may be combined together to yield the residue of the pole at $s=0$ of the expression in (26), which equals

$$
\left(\frac{Q}{\pi}\right)^{\frac{\alpha+\beta}{2}} \frac{(h, k)^{1+\alpha+\beta}}{2 h^{\frac{1}{2}+\beta} k^{\frac{1}{2}+\alpha}} Q^{2} \Phi(h k, 1) \widetilde{V}_{\alpha, \beta}(0) \widetilde{W}_{\alpha, \beta}(1) \zeta(1+\alpha+\beta) \mathcal{P}(h k ; 1,1+\alpha+\beta) .
$$

Similarly the terms $\mathcal{D}_{-\beta,-\alpha}(h, k)$ and $\mathcal{U}_{\alpha, \beta}^{2}(h, k)$ may be combined together to obtain the other main term

$$
\left(\frac{Q}{\pi}\right)^{-\frac{\alpha+\beta}{2}} \frac{(h, k)^{1-\alpha-\beta}}{2 h^{\frac{1}{2}-\alpha} k^{\frac{1}{2}-\beta}} Q^{2} \Phi(h k, 1) \widetilde{V}_{-\beta,-\alpha}(0) \widetilde{W}_{-\beta,-\alpha}(1) \zeta(1-\alpha-\beta) \mathcal{P}(h k ; 1,1-\alpha-\beta)
$$

This will complete the proof of our theorem (with the main term given in the form (10)).

We focus on establishing (68), with the proof of (67) being entirely analogous. Our goal is to match the integrand in ([66) with the corresponding integrand in (26) for $\mathcal{D}_{-\beta,-\alpha}(h, k)$. After several initial transformations, we shall move the line of integration in (66) from $\operatorname{Re}(s)=-\frac{1}{2}+\epsilon$ to $\operatorname{Re}(s)=-\epsilon$, which will then permit the sum over $c \leq C$ to be extended to infinity. Then we will obtain the desired perfect matching.

Note that

$$
\widetilde{W}_{\alpha, \beta}(1-\alpha-\beta+s)=\int_{0}^{\infty} W_{\alpha, \beta}(x) x^{1-\alpha-\beta+s} \frac{d x}{x}=\widetilde{W}_{-\beta,-\alpha}(1+s) .
$$

Next note that (using the definitions (11) and (59))

$$
\mathcal{H}\left(\frac{1}{2}-\beta+s, 1-\alpha-\beta+2 s\right) \widetilde{V}_{\alpha, \beta}(-s)=\widetilde{V}_{-\beta,-\alpha}(s) \frac{\sqrt{\pi} \Gamma\left(\frac{\alpha+\beta}{2}-s\right)}{\Gamma\left(\frac{1-\alpha-\beta}{2}+s\right)} .
$$

Using this together with the functional equation for $\zeta(s)$, we obtain

(70)

$$
\pi^{-\frac{\alpha+\beta}{2}+s} \zeta(\alpha+\beta-2 s) \mathcal{H}\left(\frac{1}{2}-\beta+s, 1-\alpha-\beta+2 s\right) \widetilde{V}_{\alpha, \beta}(-s)=\pi^{\frac{\alpha+\beta}{2}-s} \zeta(1-\alpha-\beta+2 s) \widetilde{V}_{-\beta,-\alpha}(s) .
$$

We now turn to the quantity $\mathcal{K}_{1}\left(-s, \frac{1}{2}-\beta+s,-2 s-1+\alpha+\beta ; c, h, k\right)$, which is defined through (63), (64), and (55). These definitions are all in terms of Euler products, and therefore we need only compute the relevant Euler factor for $\mathcal{K}_{1}$ at each prime $p$. This is a straightforward computation, and we give a sketch for a generic prime $p \nmid c h k$, and then content ourselves with stating the answers in the other cases. Suppose $p \nmid c h k$, and let $\mu$ 
denote the power of $p$ dividing $m$, and $\nu$ the power of $p$ dividing $n$. Then the corresponding Euler factor for $\mathcal{K}_{1}\left(-s, \frac{1}{2}-\beta+s,-2 s-1+\alpha+\beta ; c, h, k\right)$ equals

$$
\left(1-\frac{1}{p}\right)^{2}\left\{S_{1}+S_{2}+S_{3}\right\}
$$

where $S_{1}$ accounts for terms with $\mu=\nu \geq 0$ (so that $p^{\mu}$ is the power of $p$ dividing $g$ ), $S_{2}$ for terms with $\mu>\nu \geq 0$ (so that $p^{\nu}$ is the power of $p$ dividing $g$ ), and $S_{3}$ for the terms with $\nu>\mu \geq 0$ (so that $p^{\mu}$ is the power of $p$ dividing $g$ ). Recalling (55), we see that

$$
\begin{aligned}
S_{1} & =1+\frac{p^{1+2 s-\alpha-\beta}-1}{p(p-1)}+\sum_{\mu \geq 1} \frac{1}{p^{\mu(1+\alpha+\beta-2 s)}}\left(1+\frac{1}{p^{\alpha+\beta-2 s}(p-1)}-\frac{1}{p-1}\right) \\
& =\left(1+\frac{1}{p^{1+\alpha+\beta-2 s}}\right)\left(1-\frac{1}{p^{1+\alpha+\beta-2 s}}\right)^{-1}-\frac{1}{p(p-1)} .
\end{aligned}
$$

Similarly, we see that

$$
S_{2}=\left(1-\frac{1}{p^{\alpha+\beta-2 s}}\right) \sum_{\mu>\nu \geq 0} \frac{1}{p^{\mu}} \frac{1}{p^{\nu(\alpha+\beta-2 s)}}=\frac{1}{(p-1)}\left(1-\frac{1}{p^{\alpha+\beta-2 s}}\right)\left(1-\frac{1}{p^{1+\alpha+\beta-2 s}}\right)^{-1}
$$

Finally $S_{3}$ evaluates to be exactly the same as $S_{2}$. Putting all these together, we find that the Euler factor for a prime $p \nmid c h k$ equals

$$
\left(1-\frac{1}{p}\right)^{2}\left(\frac{p^{2}+p-1}{p(p-1)}\right)=\left(1-\frac{2}{p^{2}}+\frac{1}{p^{3}}\right)
$$

For a prime $p \mid c$ (and so necessarily $p \nmid h k$ ) the corresponding Euler factor is

$$
\left(1-\frac{1}{p}\right)^{2}\left(1+\frac{p^{1-\alpha-\beta+2 s}-1}{p(p-1)}\right)=\left(1-\frac{1}{p}\right)\left(1-\frac{1}{p}-\frac{1}{p^{2}}+\frac{1}{p^{1+\alpha+\beta-2 s}}\right) .
$$

Lastly, suppose $p \mid h k$ and let $\eta$ denote the power of $p$ dividing $h$, and $\kappa$ the power dividing $k$ (with $\eta+\kappa \geq 1$ ). Here the Euler factor simplifies (after a moderate amount of calculation) to give

$$
\left(1-\frac{1}{p}\right) p^{\min (\kappa, \eta)(1+2 s-\alpha-\beta)}
$$


Combining (69), (70), and the Euler factor computations of (171), (172), and (173), we may recast the integral in (66) as

$$
\begin{aligned}
& -\frac{Q^{2}}{2}\left(\frac{Q}{\pi}\right)^{-\frac{\alpha+\beta}{2}} \frac{\phi(h k)}{h k} \frac{(h, k)^{1-\alpha-\beta}}{h^{\frac{1}{2}-\alpha} k^{\frac{1}{2}-\beta}} \frac{1}{2 \pi i} \int_{\left(-\frac{1}{2}+\epsilon\right)} \zeta(1-\alpha-\beta+2 s) \widetilde{V}_{-\beta,-\alpha}(s) \widetilde{W}_{-\beta,-\alpha}(1+s) \\
& \times\left(\frac{Q}{\pi H K}\right)^{s} \sum_{\substack{c \leq C \\
(c, h k)=1}} \frac{\mu(c)}{c^{2-\alpha-\beta+2 s}} \prod_{p \nmid c h k}\left(1-\frac{2}{p^{2}}+\frac{1}{p^{3}}\right) \\
& \times \prod_{p \mid c}\left(1-\frac{2}{p}+\frac{1}{p^{3}}+\frac{1}{p^{1+\alpha+\beta-2 s}}-\frac{1}{p^{2+\alpha+\beta-2 s}}\right) \frac{d s}{s} .
\end{aligned}
$$

We move the line of integration to $\operatorname{Re}(s)=-\epsilon$, and extend the sum over $c$ to infinity incurring an error of $\ll Q^{2+\epsilon} \frac{(h, k)}{\sqrt{h k}} C^{-1}$. The extended sum over $c$ in (74) equals (writing $\gamma=1-\alpha-\beta+2 s$ for brevity)

$$
\begin{aligned}
& \sum_{(c, h k)=1} \frac{\mu(c)}{c^{1+\gamma}} \prod_{p \nmid c h k}\left(1-\frac{2}{p^{2}}+\frac{1}{p^{3}}\right) \prod_{p \mid c}\left(1-\frac{2}{p}+\frac{1}{p^{3}}+\frac{1}{p^{2-\gamma}}-\frac{1}{p^{3-\gamma}}\right) \\
= & \prod_{p \nmid h k}\left(1-\frac{2}{p^{2}}+\frac{1}{p^{3}}-\frac{1}{p^{1+\gamma}}\left(1-\frac{2}{p}+\frac{1}{p^{3}}+\frac{1}{p^{2-\gamma}}-\frac{1}{p^{3-\gamma}}\right)\right)=\mathcal{P}(h k ; 1, \gamma) .
\end{aligned}
$$

Therefore (74) becomes (note that $\Phi(h k, 1)=\phi(h k) /(h k)$ )

$$
\begin{aligned}
-\frac{Q^{2}}{2}\left(\frac{Q}{\pi}\right)^{-\frac{\alpha+\beta}{2}} \Phi(h k, 1) \frac{(h, k)^{1-\alpha-\beta}}{h^{\frac{1}{2}-\alpha} k^{\frac{1}{2}-\beta}} \frac{1}{2 \pi i} \int_{(-\epsilon)} & \zeta(1-\alpha-\beta+2 s) \widetilde{V}_{-\beta,-\alpha}(s) \widetilde{W}_{-\beta,-\alpha}(1+s) \\
& \times\left(\frac{Q}{\pi H K}\right)^{s} \mathcal{P}(h k ; 1,1-\alpha-\beta+2 s) \frac{d s}{s} .
\end{aligned}
$$

Adding this expression to the analogue of $(26)$ for $\mathcal{D}_{-\beta,-\alpha}(h, k)$, we see that their combined contribution gives precisely the residue of at $s=0$, which establishes (68).

Thus we have shown that the main terms for $\Delta_{\alpha, \beta}(h, k)$ are exactly of the shape given in the main theorem. When averaged over $h$ and $k$, the various remainder terms that arose are bounded by

$$
\sum_{h, k \leq Q^{\vartheta}} \frac{\lambda_{h} \overline{\lambda_{k}}}{\sqrt{h k}} \mathcal{E}_{h, k} \ll \frac{Q^{2+\epsilon}}{C}+C Q^{1+\vartheta+\epsilon} .
$$

Choosing finally $C=Q^{(1-\vartheta) / 2}$, the proof of the main theorem is complete.

\section{REFERENCES}

[1] Bettin, Sandro. The second moment of the Riemann zeta function with unbounded shifts. Int. J. Number Theory 6 (2010), no. 8, 1933-1944.

[2] Conrey, J. B.; Farmer, D. W.; Keating, J. P.; Rubinstein, M. O.; Snaith, N. C. Integral moments of $L$-functions. Proc. Lond. Math. Soc. 91 (2005) 33-104. 
[3] Sandro Bettin, Vorrapan Chandee, Maksym Radziwill. The mean square of the product of $\zeta(s)$ with Dirichlet polynomials. arXiv:1411.7764

[4] Balasubramanian, R.; Conrey, J. B.; Heath-Brown, D. R. Asymptotic mean square of the product of the Riemann zeta-function and a Dirichlet polynomial. J. Reine Angew. Math. 357 (1985), 161-181.

[5] Conrey, J. Brian. The mean-square of Dirichlet L-functions, arXiv:0708.2699

[6] Conrey, J. Brian; Iwaniec, H.; Soundararajan, K. Asymptotic Large Sieve, arXiv:1105.1176

[7] Conrey, J. Brian; Iwaniec, Henryk; Soundararajan, Kannan. Critical zeros of Dirichlet L-functions. J. Reine Angew. Math. 681 (2013), 175-198.

[8] Conrey, J. B.; Iwaniec, H.; Soundararajan, K. The sixth power moment of Dirichlet L-functions, Geom. and Funct. Analysis. 22, (2012), 1257-1288.

[9] Feng, Shaoji. Zeros of the Riemann zeta function on the critical line. J. Number Theory 132 (2012), no. $4,511-542$

[10] Iwaniec, H. Lectures on the Riemann zeta function. University Lecture Series, 62. American Mathematical Society, Providence, RI, 2014. viii+119 pp.

[11] Iwaniec, H.; Kowalski, E. Analytic number theory. AMS Colloquium Publications, vol. 53, 2004.

[12] Iwaniec, H.; Sarnak, P. Dirichlet L-functions at the central point. Number theory in progress, Vol. 2 (Zakopane-Koscielisko, 1997), 941-952, de Gruyter, Berlin, 1999.

[13] Selberg, Atle Contributions to the theory of Dirichlet's L-functions. Skr. Norske Vid. Akad. Oslo. I. 1946, (1946). no. 3, 62 pp.

[14] Titchmarsh, E.C. The theory of the Riemann zeta-function. Second edition, revised by D.R. HeathBrown. Oxford University Press, 1986.

[15] Young, Matthew P. The reciprocity law for the twisted second moment of Dirichlet L-functions. Forum Math. 23 (2011), no. 6, 1323-1337.

American Institute of Mathematics, San Jose, CA 95112, USA, School of Mathematics, University of Bristol, Bristol BS8 1TW, UK

E-mail address: conrey@aimath.org

Department of Mathematics, Rutgers University, Piscataway, NJ 08854, USA

E-mail address: iwaniec@math.rutgers.edu

Department of Mathematics, Stanford University, Stanford, CA 94305, USA

E-mail address: ksound@stanford.edu 